\title{
Integral sliding mode for the yaw moment control of four-wheel-drive fully electric vehicles with in-wheel motors
}

\section{Tommaso Goggia, Aldo Sorniotti* and Leonardo De Novellis}

Faculty of Engineering and Physical Sciences,

University of Surrey,

Guildford, Surrey, GU2 7XH, UK

Email: tommaso.goggia01@universitadipavia.it

Email: a.sorniotti@surrey.ac.uk

Email: 1.denovellis@surrey.ac.uk

*Corresponding author

\section{Antonella Ferrara}

Department of Electrical, Computer and Biomedical Engineering (ECBE)

University of Pavia,

Via Ferrata 5, 27100 Pavia, Italy

Email: antonella.ferrara@unipv.it

\section{Andrew Pennycott, Patrick Gruber and Ilhan Yunus}

Faculty of Engineering and Physical Sciences,

University of Surrey,

Guildford, Surrey, GU2 7XH, UK

Email: a.pennycott@surrey.ac.uk

Email: p.gruber@surrey.ac.uk

Email: i.yunus@surrey.ac.uk

\begin{abstract}
Fully electric vehicles with individually controlled motor drives allow the continuous actuation of direct yaw moment control in order to enhance vehicle safety and the handling performance by achieving a set of reference understeer characteristics. For applications on real vehicles, the control structure must provide ease of implementation, robustness and tunability. This paper discusses an integral sliding mode formulation for torque-vectoring control, which fulfils these requirements. The control structure is presented with reference to the vehicle cornering performance objectives, the motivation for integral sliding mode control and the selection of the controller parameters for stability and chattering avoidance. Six different manoeuvres are simulated for an in-wheel electric motor drivetrain layout. The results show that integral sliding mode control has significant benefits over a more conventional control method based on a combined feedforward and proportional-integral-derivative controller. The integral sliding mode controller does not require fine tuning of a feedforward control action and is characterised by superior tracking performance and disturbance rejection properties.
\end{abstract}


Keywords: integral sliding mode; torque-vectoring; yaw rate control; tracking performance; disturbance rejection; electric vehicle; in-wheel motors.

Reference to this paper should be made as follows: Goggia, T., Sorniotti, A., De Novellis, L., Ferrara, A., Pennycott, A., Gruber, P. and Yunus, I. (2015) 'Integral sliding mode for the yaw moment control of four-wheel-drive fully electric vehicles with in-wheel motors', Int. J. Powertrains, Vol. 4, No. 4, pp.388-419.

Biographical notes: Tommaso Goggia received his MSc degree in Computer Engineering (Summa Cum Laude) from the University of Pavia, Italy, in 2013. He was also a student at the Almo Collegio Borromeo of Pavia, and at the Science and Technology class of the Institute for Advanced Studies (IUSS) of Pavia. After a period as a Research Assistant at the University of Surrey, UK, he is currently working as a Software Testing Engineer at McLaren Automotive Ltd.

Aldo Sorniotti received his MSc degree in Mechanical Engineering (2001) and a PhD degree in Applied Mechanics (2005) from the Politecnico di Torino, Italy. He is a Reader in Advanced Vehicle Engineering at the University of Surrey, UK. His main research interests are in the areas of vehicle dynamics control and transmission systems for electric vehicles. He is the E-VECTOORC project coordinator.

Leonardo De Novellis received his MSc in Mechanical Engineering (2006) and $\mathrm{PhD}$ in Mechanical and Biomechanical Design (2010) from the Politecnico di Bari, Italy. From 2011 until 2014, he was a Research Fellow at the University of Surrey, UK. He is currently working as a System Testing Engineer at Brembo. His main research interests are in the areas of vehicle dynamics control and continuously variable transmissions.

Antonella Ferrara received her MSc in Electronic Engineering (1987) and PhD in Computer Science and Electronics (1992) from the University of Genova, Italy. Since 2005, she is a Full Professor of Automatic Control at the University of Pavia, Italy. Her main research interests are in the areas of sliding mode control applied to automotive systems, process control and robotics. She is a member of the IEEE Technical Committee on Automotive Control, and the Chair of the Women in Control Committee.

Andrew Pennycott received his MEng and $\mathrm{PhD}$ degrees in Mechanical Engineering from the University of Glasgow, Glasgow, UK, in 2004 and 2008, respectively. In 2012-2013, he was a Research Fellow at the University of Surrey, UK. Since 2013, he has been a Research Associate with Imperial College, UK. His research is focused on control engineering for automotive and rehabilitation applications.

Patrick Gruber received his MSc in Motorsport Engineering and Management from Cranfield University, UK, in 2005. He received his PhD in Mechanical Engineering from the University of Surrey, UK, in 2009. He is a Senior Lecturer in Advanced Vehicle Systems Engineering at the University of Surrey. His current research is in the field of tyre dynamics and in the development of novel tyre models.

Ilhan Yunus received his MSc in Automotive and Motorsport Engineering from Brunel University, UK, in 2013. Since 2014, he has been a Research Assistant at the University of Surrey, Guildford, UK. His research interests are in the area of vehicle dynamics. 


\section{Introduction}

Fully electric vehicles with individually controlled electric motor drives allow significantly improved vehicle dynamics over vehicles with equal torque on the left and right wheels and constant torque distribution between the front and rear axles. Torque-vectoring - the controlled distribution of the traction and braking torques among the wheels, also called direct yaw moment control - enables the design of the steady-state and transient cornering responses of the vehicle (De Novellis et al., 2012). This has a potential impact on vehicle behaviour with benefits regarding safety and the handling performance, more so than through the traditional approach of fine tuning hardware parameters such as mass distribution and suspension elasto-kinematics (De Novellis et al., 2013; Genta, 1997). From a terminology viewpoint, since the first torque-vectoring systems for internal-combustion-engine-driven vehicles were based on torque-vectoring differentials with very limited effect in braking, the concept of torque-vectoring was mainly associated with traction conditions. In the context of an electric vehicle with significant regeneration capability, torque-vectoring can refer to individual wheel torque control in both traction and braking.

The precise controllability of the torque generated by the electric motor drives together with their fast dynamics allows a continuous and seamless actuation of torquevectoring, which is a significant improvement over conventional stability control systems found on current production vehicles (van Zanten et al., 1995). With the conventional systems, the interventions are based on the reduction of the engine torque and the generation of friction braking torques on specific wheels (Takahashi et al., 2012). As a consequence, the activation of the stability control system reduces vehicle velocity, making the intervention practical for enhancing vehicle safety in critical transient conditions, identified when the offset between the reference value of vehicle yaw rate and the actual value of vehicle yaw rate exceeds assigned thresholds (Tseng et al., 1999; van Zanten, 2000). Alternatively, the activation of the stability control system can be triggered by an excess of estimated sideslip angle, corresponding to a potential vehicle oversteer situation in comparison with the nominal conditions.

These safety functionalities can be enhanced by means of torque-vectoring through the electric motors, as analysed in detail in De Novellis et al. (2015) through experiments: a yaw moment can be continuously generated without variation of the net traction force. However, "despite the significant volume of theoretical studies of torque-vectoring on vehicle handling control, there is no widely accepted design methodology of how to exploit it to improve vehicle handling and stability significantly" (Crolla and Cao, 2012). In this respect, within the vehicle controller implementation presented in this paper, the driver can select between different driving modes (e.g., Normal, Sport and Eco), each of these corresponding to a specific set of reference understeer characteristics, and hence to different reference yaw rates, which can enhance the driver's experience also in common driving conditions at moderate lateral accelerations.

The conventional control structure of existing industrially implemented stability control systems is based on gain-scheduled proportional-integral-derivative (PID) controllers, in which the gains are usually varied as functions of vehicle speed and the under/oversteer condition. Pastor et al. (1998) and Hartmann (2004) are two of the many relevant patents on this subject. A PID controller is effective and easily tuneable for these applications, in which the steady-state cornering response is not modified by the control system. Industrial stability control systems are rarely active and, therefore, can be quite 
abrupt in their safety-related interventions. However, for a continuously active torquevectoring controller with reference understeer characteristics significantly different from those of the vehicle without torque-vectoring, the PID contribution alone cannot provide the required precision in terms of tracking performance with satisfactory system stability, and disturbance and noise rejection properties. Therefore, for the continuous tracking of a reference yaw rate through torque-vectoring, the PID (feedback contribution) is usually integrated with a feedforward (FF) component (De Novellis et al., 2013; Pennycott et al., 2014), generating at least a static contribution. The role of the static part of the FF contribution is to provide the required understeer characteristic of the vehicle in quasistatic conditions (i.e., for slow steering wheel inputs, and any longitudinal acceleration and deceleration levels). The design of the static part of the FF controller can be based on an off-line optimisation procedure using a nonlinear vehicle model in order to achieve the reference vehicle behaviour (De Novellis et al., 2014b). An additional dynamic FF contribution (De Novellis et al., 2015; Canale et al., 2009) is useful for reducing yaw rate overshoots during transients, which are significant especially if the reference cornering behaviour has a lower degree of understeer than that of the same vehicle without torque-vectoring control. In these applications the PID controller intervenes to correct vehicle response during transients or to compensate for the unmodelled dynamics, either continuously or when pre-defined yaw rate error thresholds are exceeded. The main drawback of the described control structure is that especially the FF contribution is not robust if the actual vehicle behaviour deviates from the expected one (e.g., because of the use of different tyres or their wear, or the variation of vehicle payload).

Other authors have suggested alternative, more advanced control methodologies for direct yaw moment control than the integrated PID and FF control structure, such as second order sliding mode control (Canale et al., 2005), internal model control (Canale et al., 2009), linear quadratic regulators (Xiong et al., 2012), model predictive controllers based on conventional and explicit formulations (Chang and Gordon, 2007; Gao et al., 2014), and optimal controllers based on H-infinity and designed using linear matrix inequalities (Fallah et al., 2013; Ahn et al., 2012). There are also limited studies assessing the relative performance of different control structures. For instance, a comparison between a PID controller and an H-infinity controller for yaw rate control, designed through a single-track vehicle model and assessed through a 10 degree-of-freedom simulator, is presented in Assadian and Hancock (2005). The results show performance benefits of the $\mathrm{H}$-infinity controller, though further simulations are required for assessing the robust performance of the $\mathrm{H}$-infinity controller against the variation of physical parameters such as mass, yaw moment of inertia and shock absorber damping characteristics. Furthermore, the H-infinity control synthesis based on mixed sensitivity does not usually allow fast and empirical fine tuning of the controller parameters during vehicle testing on a proving ground, which is an essential requirement for production vehicles. In another comparison study (De Novellis et al., 2014c), the performance of a combined PID and FF yaw rate controller is contrasted with that provided by different formulations of second order sliding mode control, characterised by the absence of a discontinuity in their control action. In fact, discontinuity in the control output is the main issue preventing the industrial adoption of first order sliding mode controllers for vehicle yaw moment control. Moreover, in the existing literature the actual performance of the alternative methods for the calculation of the reference yaw moment has not been critically evaluated through extensive vehicle experiments against the conventional control methodologies industrially adopted by Tier 1 suppliers and car manufacturers. In 
conclusion, the detailed analysis of the benefits of the more advanced control techniques in terms of robustness and ease-of-tuning is still incomplete and none of those applications, with the exception of Canale et al. (2009) and more comprehensively (De Novellis et al., 2014c), has had the explicit objective of continuously altering the understeer characteristic.

The continuous tracking of a reference yaw rate significantly different from that of the vehicle with equal/constant wheel torque distribution is actually feasible only in the case of effective tyre-road friction coefficient estimation. In fact, the reference yaw rate is a function of the maximum available tyre-road friction coefficient. Moreover, also the FF yaw moment contribution, if it is present in the control structure, must be varied as a function of the estimated maximum friction coefficient. Approximate friction estimation when the vehicle reaches its limit conditions is relatively straightforward for a direct yaw moment controller designed to work in emergency conditions without comfort-related concerns, and is standard practice in existing stability control systems. For example, Eckert (1998) details a method that considers both the measured longitudinal and lateral acceleration contributions for the estimation of the friction coefficient within a stability control system actuating the friction brakes. For the same application of Eckert (1998), a recent patent (Chimner and Grogg, 2013) proposes least square estimators calculating the partial derivative of the measured yaw rate over an estimated yaw rate (corresponding to the steady-state yaw rate of a single-track vehicle model), or the partial derivative of the normalised lateral force over the steering angle. These are used as input parameters for empirically derived look-up tables providing the estimated maximum tyre-road friction coefficient for the stability control system.

However, maximum friction estimation is very difficult at the level of timeliness and precision required within a continuously active torque-vectoring system and can provoke significant actuation discontinuities, thereby causing vehicle drivability issues. In this respect, Muller et al. (2003), Ray (1997), Shim and Margolis (2004), Baffet et al. (2006), Hsu et al. (2010), Hahn et al. (2002), Ahn (2011) and Chen and Wang (2010) provide an overview of slip-based methods for tyre-road friction estimation when the tyre is operating relatively far from its friction limits. For example, Muller et al. (2003) outlines a possible method based on the online measured slope of the longitudinal force - slip ratio characteristic, which requires significant additional progress for being applied to an accurate friction identifier. Ray (1997) presents an extended Kalman-Bucy filter for the estimation of tyre tangential forces, based on a relatively complex eight-degree-of-freedom vehicle model. The model formulation does not require the inclusion of a tyre force model. The outputs of the extended Kalman-Bucy filter are used by a Bayesian hypothesis selection algorithm, which estimates the friction coefficient. The experimental results comprehensively demonstrate the good performance of the tyre force estimator; however, the friction estimator is experimentally assessed only in high friction conditions, while variable friction conditions are considered only through simulations. In Shim and Margolis (2004), a model-based friction estimation approach is proposed, in which the wheel speed and yaw rate outputs of relatively simple vehicle models are compared with the measured values of the respective parameters. Two independent PID controllers adjust the tyre friction coefficient of the simulation models in order to decrease the errors. A weighting function calculates the estimated friction coefficient, starting from the independently estimated friction coefficient values for the compensation of the wheel speed and yaw rate errors. Baffet et al. (2006) critically analyses the performance of different observers for tyre force estimation and then 
proposes a Quasi-Newton method, independent from the tyre force and sideslip observer, to calculate the Burckhardt tyre model coefficients, functions of the friction coefficient, in order to minimise the lateral force error between the Burckhardt model and the observer output. The rather negative conclusion, based on experiments, is that "when lateral dynamics are small (sideslip angle lower than 4 deg.) there are not sufficiently significant values to identify tyre-road friction curves". Very importantly, Hsu et al. (2010) points out the effectiveness of the pneumatic trail, which can be estimated starting from the normally available steering system torques, for tyre-road friction coefficient estimation well before the friction limits are reached. In particular, Hsu et al. (2010) adopts the prototype P1 vehicle for experimentally demonstrating the effectiveness of a nonlinear observer based on the steering system for predicting the friction limits "once the tyres have utilised only $50 \%$ of their maximum lateral force", which is more than sufficient for the specific application of the direct yaw moment control for fully electric vehicles discussed in this paper. Hahn et al. (2002) discusses a GPS-based real-time identification algorithm of tyre-road friction coefficient, which does not require large longitudinal and lateral slips for reliable estimates, and shows a parameter convergence rate of $1 \mathrm{~s}$. The main limitation relates to the possible lack of satellite visibility, for example in urban driving. Finally, De Novellis et al. (2014c) presents an integrated yaw rate and sideslip angle yaw moment controller for electric vehicles with multiple motors, in which the sideslip contribution is activated only in case of erroneous friction coefficient estimation, and hence excessive reference yaw rate. In conclusion, the existing literature demonstrates that continuously active yaw rate-based algorithms are actually safely implementable for a variety of friction conditions if they are coupled with the most advanced state estimators, such as those in Ray (1997), Hsu et al. (2010) and Hahn et al. (2002), or sideslip emergency controllers (De Novellis et al., 2014c).

Integral sliding mode (ISM), the focus of this paper, has a number of advantages compared to conventional sliding mode formulations (first or second order sliding mode), which provide robustness once the system reaches the sliding manifold. With ISM, the order of the motion equation is equal to the order of the equation of the original system and the sliding mode can thus begin from the first sampling instant. Moreover, this particular sliding mode formulation prevents chattering and discontinuities of the control action - a major disadvantage of some sliding mode control realisations (Utkin et al., 1999 ) - through the inclusion of a first order filter on its output. ISM is usually presented as a disturbance observer, as it includes another continuous controller designed for the system operating in its nominal conditions. The ISM controller provides robustness to the nominal controller, which is usually selected to be easy-to-tune and can be characterised by a simple formulation. As a consequence, the sliding mode control structure combines the smooth control action and robustness of higher order sliding mode (Utkin et al., 1999) with the tunability of the industrial controllers.

In this paper, a robust yaw moment controller formulation based on ISM incorporating a constant gain PID controller is presented, with the general purpose of continuously tracking reference understeer characteristics significantly different from those of the vehicle without the controller. The procedure for tuning the parameters of the ISM controller based on system uncertainty and chattering prevention is detailed. The paper includes a comparison of the performance of the proposed integral sliding mode controller with a combined PID FF controller and the baseline vehicle (without any controller) in terms of vehicle response in nominal conditions and when uncertainties and disturbances are considered. 


\section{Vehicle model for controller performance evaluation}

The control structure performance is assessed with vehicle dynamics simulations performed with a CarMaker model (IPG Automotive, 2015), which considers sprung and unsprung mass dynamics and suspension elasto-kinematics. Tyres are modelled with the Pacejka Magic Formula with variable relaxation length (Pacejka, 2006). The chassis model (including sprung and unsprung masses, steering system, suspension elasto-kinematics and tyres) has been extensively validated against experimental results obtained during different sessions at the Lommel proving ground (Belgium) under steady-state and transient conditions (skid pads and step steers), including the adoption of a steering robot for enhancing the repeatability of the experiments (De Novellis et al., 2013, 2014a, 2015).

The electric drivetrains are modelled in MATLAB Simulink and consist of in-wheel motors connected to a single-speed transmission. The efficiency of the drivetrain components (i.e., motor, inverter and transmission) is included through maps, which are functions of torque, speed and temperature. The electro-hydraulic braking system unit for the independent pressure modulation of the friction brakes and the decoupling between brake pedal force and calliper actuation is modelled in MATLAB Simulink according to the approach described in De Novellis et al. (2015). The lumped-parameter battery model is based on the equivalent circuit approach outlined in Gao et al. (2002). The main vehicle parameters, referring to a case study sports utility vehicle, are reported in Table 1. The Simulink part of the model also includes the definition of the driver's inputs (steering wheel angle, accelerator pedal position and brake pedal position) and runs in co-simulation with the CarMaker chassis model.

Table 1 Main vehicle parameters for the simulation study

\begin{tabular}{lcc}
\hline Symbol & Description & Quantity \\
\hline$m$ & Vehicle mass & $1,797 \mathrm{~kg}$ \\
$J_{z}$ & Yaw moment of inertia & $2,995 \mathrm{kgm}^{2}$ \\
$a$ & Front semi-wheelbase & $1.05 \mathrm{~m}$ \\
$b$ & Rear semi-wheelbase & $1.59 \mathrm{~m}$ \\
$T_{F}$ & Front track width & $1.63 \mathrm{~m}$ \\
$T_{R}$ & Rear track width & $1.64 \mathrm{~m}$ \\
$h_{C G}$ & Height of the centre of gravity & $0.66 \mathrm{~m}$ \\
$P_{M, M A X}$ & Maximum drivetrain power (combined value for the 4 drivetrains) & $320 \mathrm{~kW}$ \\
\hline
\end{tabular}

\section{Vehicle controllers}

\subsection{Overall control structure}

A simplified schematic of the torque-vectoring controller is shown in Figure 1. The drivability controller generates an overall reference traction/braking torque, $T_{w, t o t}$, mainly based on the accelerator and brake pedal positions, $a_{p p}$ and $b_{p p}$, respectively. A reference yaw rate, $r_{r e f}$, is calculated based on the steering wheel angle, $\delta$, vehicle speed, $v$, longitudinal acceleration, $a_{x}$, and estimated friction coefficient, $\mu$. Each driving mode 
corresponds to a reference set of understeer characteristics parameterised through the following formula, which fits closely to experimental results:

$$
a_{y}= \begin{cases}\frac{1}{K_{U}^{l i n}\left(a_{x}, \mu\right)} \delta_{W, d y n} & \text { if } \delta_{W, d y n}<a_{y}^{*}\left(a_{x}, \mu\right) K_{U}^{\operatorname{lin}}\left(a_{x}, \mu\right) \\ a_{y, M A X}\left(a_{x}, \mu\right)+\left(a_{y}^{*}\left(a_{x}, \mu\right)-a_{y, M A X}\left(a_{x}, \mu\right)\right) & \text { if } \delta_{W, d y n} \geq a_{y}^{*}\left(a_{x}, \mu\right) K_{U}^{\operatorname{lin}}\left(a_{x}, \mu\right) \\ \frac{\left.K_{U}^{\operatorname{lin}}\left(a_{x}, \mu\right)\right)_{y}^{*}\left(a_{x}, \mu\right)-\delta_{W, d j n}}{\left(a_{y, M A X}\left(a_{x}, \mu\right)-a_{y}^{*}\left(a_{x}, \mu\right)\right) K_{U}^{(i \operatorname{lin}}\left(a_{x}, \mu\right)} & \end{cases}
$$

A typical example of understeer characteristic for conditions of constant velocity will be discussed in Figure 4 (Section 4.1), while other examples are shown in De Novellis et al. (2014b). Based on equation (1), the reference understeer characteristics can be expressed through a set of parameters with a clear physical meaning, namely:

$1 K_{U}^{l i n}\left(a_{x}, \mu\right)$, reference value of the understeer gradient in the linear region of the understeer characteristic

$2 a_{y}^{*}\left(a_{x}, \mu\right)$, reference value of the maximum lateral acceleration in the linear region of the understeer characteristic

$3 a_{y, \text { MAX }}\left(a_{x}, \mu\right)$, reference value of the maximum lateral acceleration of the vehicle.

The tables of the steady-state reference yaw rate, $r_{r e f, S}=r_{\text {ref }, S}\left(\delta, v, a_{x}, \mu\right)$, are computed off-line, starting from the reference understeer characteristics, according to the following four steps:

1 For the assigned values of $a_{y}$ and $v$, the steady-state reference yaw rate is defined as $r_{\text {ref }, S}=\frac{a_{y}}{v}$, with the hypothesis of $\dot{\beta} \approx 0$, relating to steady-state cornering conditions.

2 The corresponding dynamic steering angle (at the wheel), $\delta_{W, d y n}$, is expressed by using equation (1), associated with the reference understeer characteristics: $\delta_{W, d y n}=$ $\delta_{W, d y n}\left(a_{x}, a_{y}, \mu\right)$.

3 The actual steering angle (at the wheel), including the dynamic and kinematic contributions, is computed as:

$$
\begin{aligned}
\delta_{W} & =\delta_{W}\left(a_{y}, v, a_{x}, \mu\right)=\delta_{W, d y n}\left(a_{y}, a_{x}, \mu\right)+\delta_{W, k i n} \\
& =\delta_{W, d y n}\left(a_{y}, a_{x}, \mu\right)+L \frac{a_{y}}{v^{2}} \\
& =\delta_{W, d y n}\left(r_{r e f, S}, v, a_{x}, \mu\right)+L \frac{r_{r e f, S}}{v}
\end{aligned}
$$

4 The steering wheel angle, $\delta$, corresponding to $\delta_{W}$, is calculated through the nonlinear map describing the steering system kinematics: $\delta=\delta\left(\delta_{W}\right)$. The tables of $r_{r e f, S}=r_{r e f, S}\left(\delta, v, a_{x}, \mu\right)$, actually implemented in the real-time controller, provide the steady-state reference for the control system. 
The results discussed in this paper refer to the understeer characteristics of the Sport driving mode, characterised by a reduced level of understeer in comparison with the vehicle without the torque-vectoring controller. Note that in the Sport driving mode the steady-state and transient objectives for the controlled vehicle tend to conflict with each other. In fact, the reduction in steady-state understeer through torque-vectoring in the Sport mode provokes an increase in the steady-state absolute value of sideslip angle $\beta$. This aspect can be a requirement of the car manufacturer in order to provide a sport-oriented vehicle feel. However, the torque-vectoring controller must also be capable of limiting the overshoots and peaks typical of sideslip response in transient conditions. These overshoots and consequent peaks tend to be higher for vehicles with a reduced level of understeer, typical of the controlled vehicle in Sport mode. In summary, the torque-vectoring controller should reduce vehicle stability in steady-state conditions in order to improve the handling performance and the ease-to-drive aspects - but, at the same time, it should be able to increase stability during transients.

Corresponding to Figure 1, within the reference yaw rate generator, the reference yaw rate, $r_{r e f}$, is calculated starting from the signal, $r_{r e f, S}$, produced by the off-line computed look-up table, through the first order transfer function: $r_{r e f}=r_{r e f, S} \frac{1 / \tau_{r}}{p+1 / \tau_{r}}$. In order to examine the stability performance of the ISM controller with a very aggressive tuning of the reference yaw rate, $\tau_{r}=0$ is used in the simulations reported here (i.e., the filter is deactivated). The reference yaw rate is compared with the actual yaw rate, $r$, and the resulting yaw rate error, $-\Delta r=r_{r e f}-r$, is sent to the high-level controller, which is the focus of this paper. The high-level controller calculates the reference yaw moment, $M_{z, \text { CTRL}}$, and the overall wheel torque, $T_{w, \text { tot,mod. }}$ For $|\Delta r|<\left|\Delta r_{\text {threshold }}\right|$, $T_{w, t o t, \text { mod }}=T_{w, \text { tot }}$, as in normal driving conditions the torque-vectoring controller produces $M_{z, C T R L}$ without modifying the driver torque demand. Analogously to existing stability control systems, if $|\Delta r| \geq\left|\Delta \mathrm{r}_{\text {threshold }}\right|$, an emergency condition is identified and the overall wheel torque demand is modified (reduced) by the high-level controller, according to the principles outlined in Takahashi et al. (2012).

Figure 1 Simplified schematic of the overall control structure

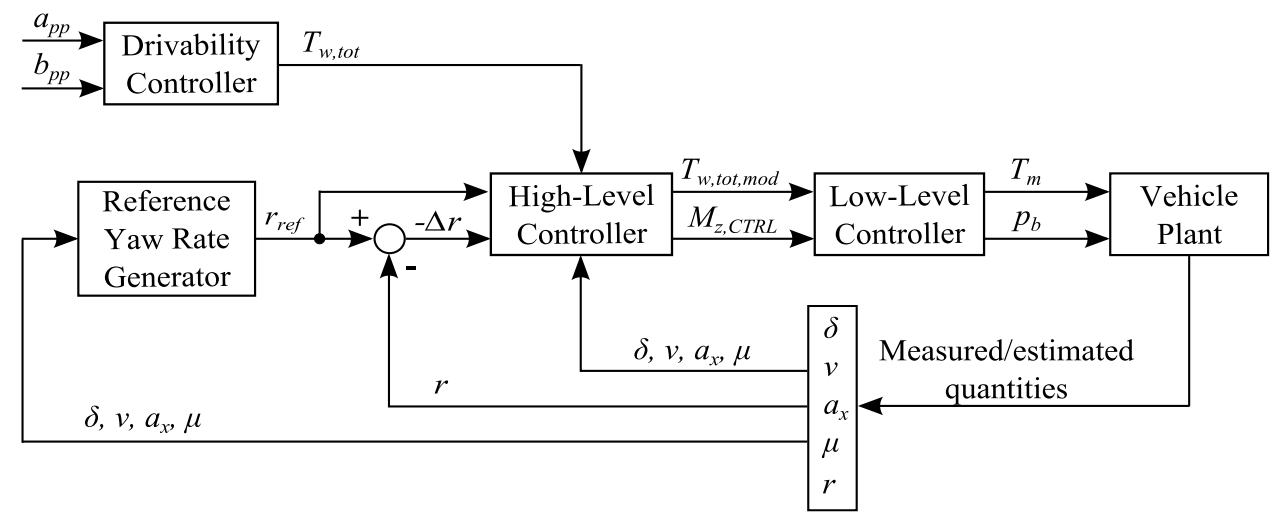

The low-level controller generates the individual reference torques, $T_{m}$, for the electric motor drives and the reference friction brake pressures, $p_{b}$. This can be performed according to optimisation-based approaches (Tjonnas et al., 2010; Naraghi et al., 2010) or 
rule-based criteria (Tanaka et al., 1992). As this article focuses on the analysis of the high-level controller formulation, a simple rule-based algorithm is used, where $T_{m}$ is derived from the reference wheel torques, $T_{w}$, calculated via the equations:

$$
\left\{\begin{array}{l}
T_{w, R F / L F}=\frac{T_{w, t o t, m o d}}{2} \tau_{T, F} \pm \frac{M_{z, C T R L}}{T_{F}} \tau_{M, F} R_{w} \\
T_{w, R R / L R}=\frac{T_{w, t o t, \text { mod }}}{2}\left(1-\tau_{T, F}\right) \pm \frac{M_{z, C T R L}}{T_{R}}\left(1-\tau_{M, F}\right) R_{w} .
\end{array}\right.
$$

The non-dimensional coefficients, $\tau_{T, F}$ and $\tau_{M, F}$ (ranging between 0 and 1 ), respectively define the front-to-total wheel torque and yaw moment distributions. In this study, $\tau_{T, F}=\tau_{M, F}=0.5$ in traction conditions and $\tau_{T, F}=\tau_{M, F}=0.75$ in braking conditions, with a progressive transition (defined through a look-up table) between the two values for low values of $\left|a_{x}\right|$.

Then, the reference motor torque, $T_{m}$, for a given wheel is:

$$
T_{m}=\min \left(\frac{\left|T_{w}\right|}{\tau_{\text {trans }}} ;\left|T_{m, M A X}\right|\right) \operatorname{sign}\left(T_{w}\right) .
$$

Should a wheel torque be negative, the electro-hydraulic friction brakes provide the difference between the brake torque demand and the wheel torque corresponding to the maximum motor torque for the specific operating condition (motor torque saturated at the peak or nominal level depending on the thermal condition of the electric motor drive):

$$
p_{b}=\frac{T_{m} \tau_{\text {trans }}-T_{w}}{K_{b}}
$$

\subsection{The ISM controller}

\subsubsection{General ISM formulation}

According to the ISM control formulation (Utkin et al., 1996), for a system characterised by the structure

$$
\dot{x}=f(x)+n(x) u+h(x, t)
$$

a sliding surface, $s$, consisting of two contributions can be defined as:

$$
s=s_{0}+z
$$

in which the term $s_{0}$ follows the usual definition of a sliding surface based on the states of the system. For example, in this particular application, $s_{0}$ is the opposite of the yaw rate error: $s_{0}=\Delta r=r-r_{r e f}$. The additional term, $z$, specific to ISM can be obtained by integration of

$$
\dot{z}=-\frac{d s_{0}}{d x}\left[f(x)+n(x) u-n(x) u_{1}\right], \text { with } z(0)=-s_{0}(x(0))
$$

where $u_{1}$ is the output of the discontinuous part of the sliding mode controller, that is

$$
u_{1}=-K(x) \operatorname{sign}(s) \text {. }
$$


In order to guarantee system stability according to the Lyapunov criterion, system uncertainties $h(x, t)$ must be bounded; $\mid h(x, t)) \mid<h_{\max }(x, t)$ and the following condition on $K(x)$ must be met:

$$
K(x)>\left|\frac{h_{\max }(x, t)}{n(x)}\right| .
$$

In general, the overall control action of ISM is given by

$$
u=u_{0}+u_{1, \text { filtered }}
$$

with $u_{0}$ being the output of a usually simple continuous controller, i.e., the nominal controller mentioned in Section 1, which is a PID contribution without gain scheduling in this application. $u_{1, \text { filtered }}$ is the filtered signal of $u_{1}$ and it is defined such that

$$
\tau_{\text {controller }} \dot{u}_{1, \text { filtered }}+u_{1, \text { filtered }}=u_{1} .
$$

The PID within the ISM was not coupled with a FF contribution in order to demonstrate the benefits of the ISM structure itself. The FF is usually required for the effective continuous and smooth implementation of torque-vectoring with a reference yaw rate significantly different from the steady-state one of the passive vehicle. The incorporation of a simple PID without FF allows an acceptable (but not particularly good) tracking performance, since the tuning of the gains for the specific application has to be very conservative in order to prevent noise, vibration and harshness problems in the actual implementation of the controller on a vehicle.

In general, for continuous yaw moment control a sub-optimal yaw rate tracking performance with a smooth control action is preferable over an optimal tracking performance with a control output compromising vehicle drivability. These subtle aspects can be assessed mainly by the test drivers and vehicle passengers during the experimental tests, as it is also mentioned in the conclusions of Kaiser et al. (2014): torque-vectoring "creates a feedback to the driver... and leads to an oscillating steering behaviour, which was not appreciated by the test drivers". For these reasons, the values of the PID gains used here were constrained to be particularly low (conservative design of the controller), i.e., in a range providing acceptable drivability during the experimental tests on the vehicle demonstrator of the EU FP7 project E-VECTOORC (http://www.e-vectoorc.eu). As the E-VECTOORC demonstrator has an on-board drivetrain layout, the gains suitable for that vehicle surely provide acceptable drivability for a vehicle with in-wheel motors (while the converse is not valid). The PID gains (which produce $u_{0}$ ) were tuned with a constrained particle swarm optimisation (De Novellis et al., 2015; Gaing, 2004; Lin et al., 2009) applied to a nonlinear single-track vehicle model (for the first iterations of the optimisation) and the CarMaker simulation model (for the final iterations of the optimisation). During the process, the typical properties of the feedback control system in the frequency domain, such as gain margin, phase margin, disturbance rejection and tracking bandwidth, were investigated through the linear single-track vehicle model for different linearisation points in terms of cornering stiffness of the front and rear axles. 


\subsubsection{Specific ISM formulation for torque-vectoring control}

The control formulation can be based on the yaw moment balance equation of the vehicle (Figure 2), which is

$$
\begin{aligned}
J_{z} \dot{r} & =\left(-F_{y, R F} \sin \delta_{w, R F}+F_{y, L F} \sin \delta_{w, L F}+F_{x, R F} \cos \delta_{w, R F}-F_{x, L F} \cos \delta_{w, L F}\right) \frac{T_{F}}{2} \\
& +\left(F_{y, R F} \cos \delta_{w, R F}+F_{y, L F} \cos \delta_{w, L F}+F_{x, R F} \sin \delta_{w, R F}+F_{x, L F} \sin \delta_{w, L F}\right) a \\
& -\left(F_{y, R R}+F_{y, L R}\right) b+\left(F_{x, R R}-F_{x, L R}\right) \frac{T_{R}}{2}-M_{s a, R F}-M_{s a, L F}-M_{s a, R R}-M_{s a, L R} .
\end{aligned}
$$

In this simplified model for control system design, the output of the torque-vectoring controller, $M_{z, C T R L}$, is the yaw moment contribution due to the longitudinal tyre forces.

Figure 2 Vehicle schematic (top view)

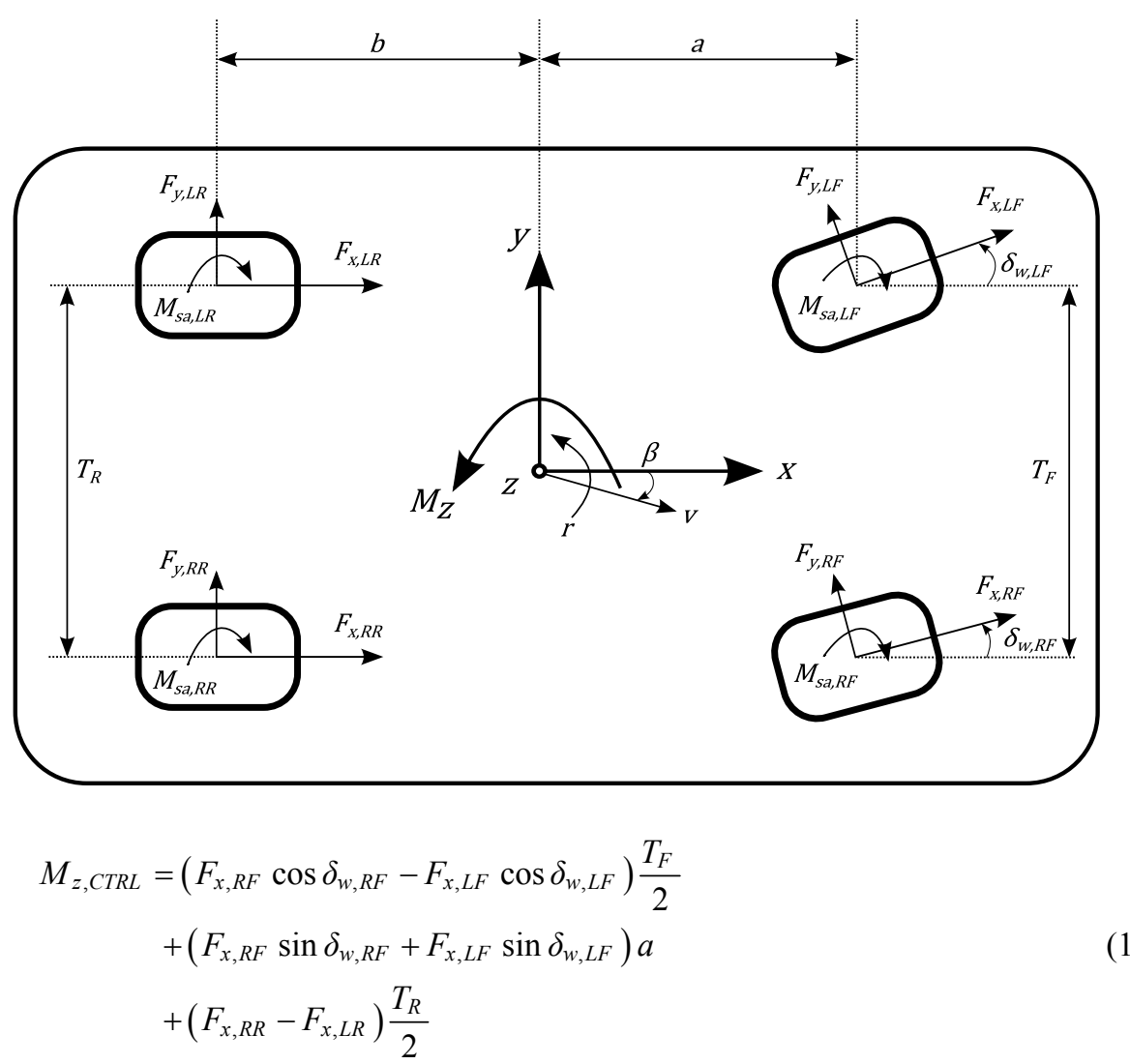

In practice, the approximation of the actuation system (consisting of the electric drivetrains and the friction brakes) will cause some discrepancy between the left and right-hand sides of equation (14). By defining 


$$
\begin{aligned}
k(r, \beta, \delta)= & \frac{1}{J_{z}}\left[\left(-F_{y, R F} \sin \delta_{w, R F}+F_{y, L F} \sin \delta_{w, L F}\right) \frac{T_{F}}{2}\right. \\
& +\left(F_{y, R F} \cos \delta_{w, R F}+F_{y, L F} \cos \delta_{w, L F}\right) a \\
& \left.-\left(F_{y, R R}+F_{y, L R}\right) b-M_{s a, R F}-M_{s a, L F}-M_{s a, R R}-M_{s a, L R}\right]
\end{aligned}
$$

and considering the additional yaw moment, $M_{z, u n c}$, due to the disturbances and uncertainties (for example, due to the bank angle of the road, lateral wind or any variation of vehicle parameters), equation (13) becomes

$$
\dot{r}=k(r, \beta, \delta)+\frac{1}{J_{z}} M_{z, \text { CTRL }}+\frac{1}{J_{z}} M_{z, \text { unc }} .
$$

As it includes terms related to lateral tyre forces, $k$ is a function of $r, \beta$ and $\delta$, as tyre slip angles depend on those kinematic parameters, and (in a second approximation) the longitudinal slip ratios, because of the interdependence between lateral and longitudinal tyre forces. The mathematical expression for $k(r, \beta, \delta)$ can be easily derived from a single-track vehicle model (Milliken and Milliken, 1995). The interaction between longitudinal and lateral tyre forces is neglected in the ISM controller design, and so the controller must be sufficiently robust to cope with the resulting errors incurred due to this approximation.

By subtracting the reference value of yaw acceleration, $\dot{r}_{r e f}$, from the left and right hand sides of equation (16), the time derivative of the yaw rate error is

$$
\Delta \dot{r}=\dot{r}-\dot{r}_{r e f}=k(r, \beta, \delta)-\dot{r}_{r e f}+\frac{1}{J_{z}} M_{z, \text { CTRL }}+\frac{1}{J_{z}} M_{z, u n c}
$$

where $M_{z, C T R L}$ is calculated from $M_{z, T O T}$, to which a saturation condition is applied according to the drivetrain torque limitations and the estimated tyre-road friction coefficient (the full equations are omitted here for brevity) in order to generate the reference yaw moment. $M_{z, T O T}$ follows the general form of the ISM controller of equation (11):

$$
M_{z, T O T}=M_{z, I S M, P I D}+M_{z, I S M, \text { filtered }}
$$

$M_{z, I S M \text {,filtered }}$ derives from $M_{z, I S M}$, through the first order filter reported in equation (12).

$$
\left.M_{z, I S M}=-J_{z} K \operatorname{sign}(s), \text { with } K>\left|h_{\max }\right|\right)
$$

As the value of the gain $K$ of the ISM must be designed taking system uncertainties into account, three different cases can be distinguished. The three cases differ in terms of the states which can be reliably estimated during vehicle operation.

Case 1 The term $k(r, \beta, \delta)$ is assumed to be unknown (no state-observer is implemented on the vehicle) and the term $\dot{r}_{\text {ref }}$ is known, hence $f=-\dot{r}_{\text {ref }}$ and $h=\frac{1}{J_{z}} M_{z, u n c}+k(r, \beta, \delta)$. In this case, $f$ is a function of the inputs $\left(\delta, a_{p p}, b_{p p}\right.$, etc.) rather than the states. The sliding mode equation is 


$$
\begin{aligned}
\dot{z} & =-\frac{d s_{0}}{d(\Delta r)}\left[-\dot{r}_{r e f}+\frac{1}{J_{z}}\left(M_{z, C T R L}-M_{z, I S M}\right)\right] \\
& =\dot{r}_{r e f}-\frac{1}{J_{z}}\left(M_{z, C T R L}-M_{z, I S M}\right) .
\end{aligned}
$$

Case 2 The whole term $k(r, \beta, \delta)-\dot{r}_{\text {ref }}$ is considered to be unknown. Hence, $f=0$ and, as a consequence, $h=\frac{1}{J_{z}} M_{z, u n c}+k(r, \beta, \delta)-\dot{r}_{r e f}$ and the sliding mode equation is$$
\dot{z}=-\frac{d s_{0}}{d(\Delta r)}\left[0+\frac{1}{J_{z}}\left(M_{z, C T R L}-M_{z, I S M}\right)\right]=-\frac{1}{J_{z}}\left(M_{z, C T R L}-M_{z, I S M}\right) .
$$

Case 3 The whole term $k(r, \beta, \delta)-\dot{r}_{r e f}$ is known with some approximation, for example, through a state estimator so that $h=\frac{1}{J_{z}} M_{z, \text { unc }}$ and the sliding mode equation is

$$
\begin{aligned}
\dot{z} & =-\frac{d s_{0}}{d(\Delta r)}\left[k(r, \beta, \delta)-\dot{r}_{r e f}+\frac{1}{J_{z}}\left(M_{z, C T R L}-M_{z, I S M}\right)\right] \\
& =-k(r, \beta, \delta)+\dot{r}_{r e f}-\frac{1}{J_{z}}\left(M_{z, C T R L}-M_{z, I S M}\right) .
\end{aligned}
$$

Figure 3 Time history of $J_{z} h(t)$ for Case 2 during a sequence of step steers at $90 \mathrm{~km} / \mathrm{h}$

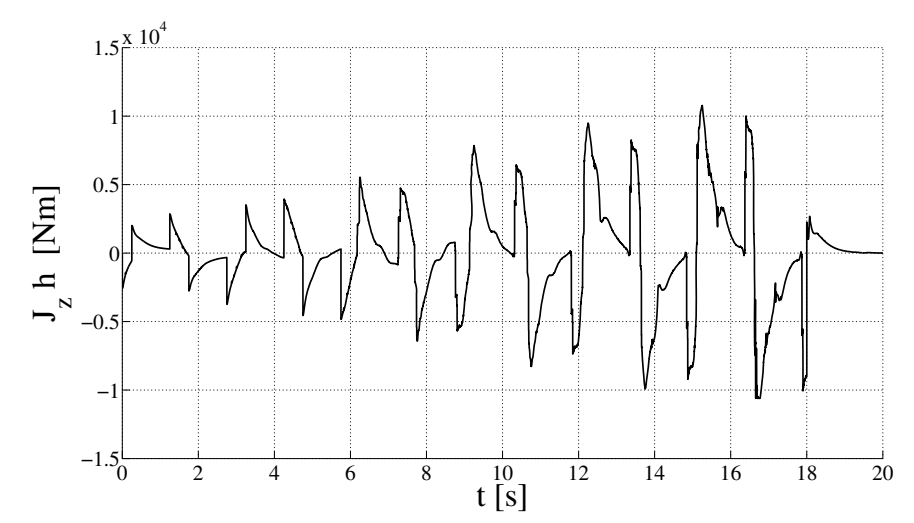

The values of $h_{\max }$ for the three cases and the subsequent values of the gain $K$ in equation (19) were estimated through vehicle dynamics simulations of the controlled vehicle during critical manoeuvres in transient conditions, including external disturbances (i.e., lateral wind) and vehicle parameter values (i.e., mass $m$ and the yaw moment of inertia $J_{z}$, which depend on the vehicle payload) different from the nominal case. For example, Figure 3 plots $J_{z} h(t)=J_{z} k(r, \beta, \delta)-J_{z} \dot{r}_{\text {ref }}$ for Case 2 during a sequence of step steers with $M_{z, u n c}=0$. From the analysis carried out in different manoeuvres, the peaks of 
$h(t)$ are approximately similar for Case 1 and Case 2 and they are marginally lower for Case 3. Based on this, it was possible to select a constant value of $J_{z} K=15,000 \mathrm{Nm}$.

The time constant $\tau_{\text {controller }}$ of equation (12) must be tuned in order to prevent any form of chattering [which is an undesired vibration of the system around the sliding manifold (Utkin et al., 1999)] and discontinuity of the control action. In particular, owing to the application of a continuously active torque-vectoring controller, the discontinuity of the control action must be avoided for comfort-related reasons. In contrast, in power electronics applications of ISM, a discontinuous control action does not represent a problem (the switching of the control action is a typical characteristic of ideal sliding mode) provided that the system trajectory actually remains on the sliding manifold without chattering.

Chattering in sliding mode controllers is usually due to the unmodelled dynamics of the actuators. In this application, the in-wheel drivetrains are characterised by very fast dynamics. In fact, the absence of half-shafts prevents any torsional oscillations at the relatively low frequencies relevant to vehicle drivability (Bottiglione et al., 2012). As a consequence, during traction the main possible source of chattering is represented by the tyre dynamics due to the longitudinal relaxation length, $L_{r e l}$ (Pacejka, 2006). These dynamics are represented by a first order transfer function with time constant $\tau_{\text {tyre }} \cong \frac{L_{r e l}}{v}$, and are not very significant at large vehicle speeds such as those of the manoeuvres investigated here. If we suppose $L_{r e l}=0.3 \mathrm{~m}$ and $v=30 \mathrm{~m} / \mathrm{s}, \tau_{\text {tyre }}=0.01 \mathrm{~s}$, whilst the natural frequency of the yaw motion [which should be the frequency of the equivalent control action of the ISM (Utkin and Shi, 1996)] is usually between 1 and $2 \mathrm{~Hz}$ for typical passenger cars. Nevertheless, despite chattering not being an issue for the specific electric drivetrain layout (but it could be in the case of on-board electric drivetrains), particular care must be devoted to smoothing the control action, so that the parameter $\tau_{\text {controller }}(=0.05 \mathrm{~s}$ for the results presented in this paper) generates a control output without oscillations perceivable by the passengers.

\subsection{The PID and FF controller}

The performance of the ISM controller is compared with that of a conventional torque-vectoring controller consisting of a combination of feedforward and PID contributions (indicated as PID FF in the remainder of the paper). The yaw moment is calculated according to

$$
M_{z, T O T}=M_{z, P I D}+M_{z, F F}\left(\delta, v, a_{x}, \mu\right) .
$$

The FF part of the controller is tuned through an offline optimisation procedure based on a separate quasi-static model developed by the authors (De Novellis et al., 2013, 2014b) in order to track a reference set of understeer characteristics, for example, defined through equation (1). The procedure generates a multi-dimensional look-up table of $M_{z, F F}$ as a function of $\delta, v, a_{x}$ and $\mu$. The purpose of the FF contribution is to achieve good tracking performance of the reference yaw rate in steady-state for nominal conditions of the vehicle. This can be accomplished provided that the actual vehicle has a cornering response and a control allocation algorithm of the wheel torques [for instance, that expressed by equation (3)] very close to that of the quasi-static model on which the optimisation is based. The quasi-static vehicle model used in the optimisation was 
developed under the assumption that the time derivatives of the main state variables of the system (vehicle sideslip angle, roll angle and tyre longitudinal slip, but not longitudinal acceleration) are zero. The procedure allows the required yaw moment for a given understeer condition and vehicle state to be determined. The resulting magnitude of $M_{z, F F}$ can be significant even in steady-state conditions, for example, if the controller is designed to radically alter the understeer characteristic of the passive vehicle, as is the case for the results presented here.

\section{Simulation results}

The ISM yaw moment controller, the PID FF controller, the PID controller (with the PID gains being the same for the three controller implementations) and the baseline vehicle are compared using simulations with the model presented in Section 2. Six manoeuvres are considered in the analysis:

1 A ramp steer manoeuvre, consisting of rotating the steering wheel at $10 \mathrm{deg} / \mathrm{s}$ at a vehicle speed $v=90 \mathrm{~km} / \mathrm{h}$. In order to keep $v$ constant, this manoeuvre implies a significant increase of torque demand to compensate the additional resistance due to lateral tyre slip (ISO 4138:2012, 2012).

2 A sequence of step steers in high friction conditions, i.e., a friction coefficient of 1.0, consisting of a sequence of steering wheel inputs in opposite directions at progressively increasing amplitude at $v=90 \mathrm{~km} / \mathrm{h}$ in order to excite the yaw dynamics of the vehicle (manoeuvre loosely based on ISO 7401:2011, 2011).

3 A sine-with-dwell test, according to the procedure defined in FMVSS 126 and ECE R13H (National Highway Traffic Safety Administration FMVSS 126, 2007).

4 A sequence of step steers in low friction conditions, i.e., a friction coefficient of 0.3.

5 A straight-ahead manoeuvre (i.e., with zero steering wheel angle) at a vehicle speed of $90 \mathrm{~km} / \mathrm{h}$, in which a sinusoidal disturbance yaw moment (i.e., a so called matched disturbance) is imposed with amplitude 1,000 Nm and frequency $3 \mathrm{rad} / \mathrm{s}$.

6 Straight-ahead manoeuvre (i.e., with zero steering wheel angle) with the vehicle subject to a lateral wind gust while it is travelling at a constant speed of $90 \mathrm{~km} / \mathrm{h}$. The speed of the lateral wind gust is $72 \mathrm{~km} / \mathrm{h}$ and is applied for $2 \mathrm{~s}$.

Two parameters are used for evaluating the tracking performance of the controllers and the degree of control effort in the most significant cases; these are the integral of the absolute value of the yaw rate error $(I A E)$ and the integral of the absolute value of the control action $(I A C A)$ given by

$$
\begin{aligned}
& I A E=\frac{1}{t_{\text {man }, \text { fin }}-t_{\text {man }, \text { in }}} \int_{t_{\text {man }, \text { in }}}^{t_{\text {man ffin }}}\left|r(t)-r_{\text {ref }}(t)\right| d t \\
& I A C A=\frac{1}{t_{\text {man }, \text { fin }}-t_{\text {man }, \text { in }}} \int_{t_{\text {man }, \text { in }}}^{t_{\text {man }, \text { fin }}}\left|M_{z, \text { CTRL }}(t)\right| d t .
\end{aligned}
$$

With regard to the simulation study, unless otherwise specified, the ISM formulation refers to Case 1 of Section 3.2.2, i.e., a simple ISM implementation without any form of 
state estimation, for the specific purpose of assessing the ISM performance in the most critical conditions.

\subsection{Ramp steer manoeuvre}

Figure 4 shows the overlap of the understeer characteristics of the passive vehicle and the controlled vehicle in the Sport driving mode in conditions of constant velocity. As expected for this driving mode, the torque-vectoring controlled vehicle is characterised by a lower value of the understeer gradient and a higher value of the maximum achievable lateral acceleration, $a_{y}$.

Figure 4 Understeer characteristics for the passive vehicle (Baseline) and the controlled vehicle in the sport-oriented driving mode (Sport)

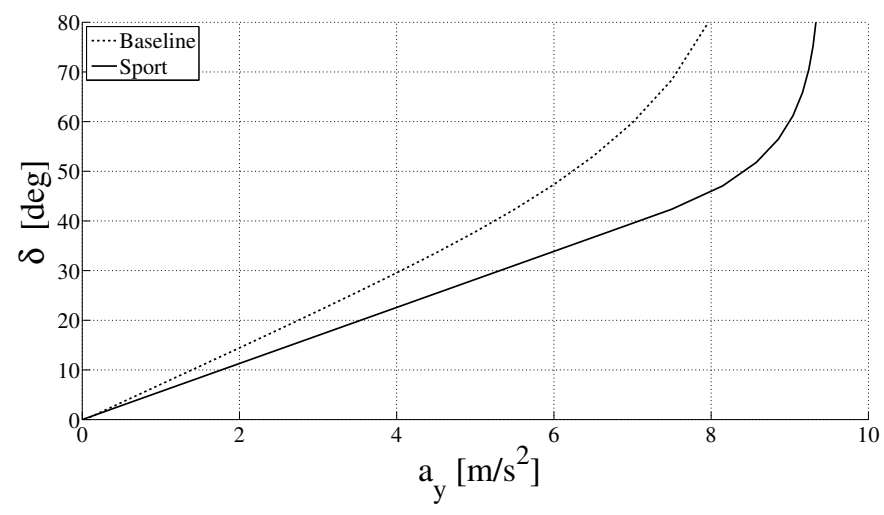

Figure 5 Top: time history of the sliding surface $s$ during the ramp steer manoeuvre for the vehicle with the ISM controller; bottom: yaw rate error, $-\Delta r$, for the passive vehicle (Baseline), the PID FF-controlled vehicle and the ISM-controlled vehicle
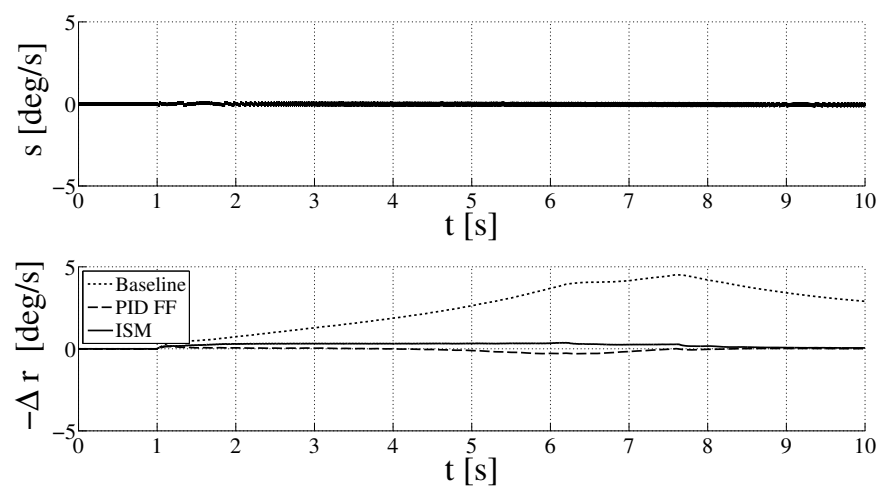
Figure 6 Yaw moment contributions during the ramp steer manoeuvre for the ISM and PID FF controllers

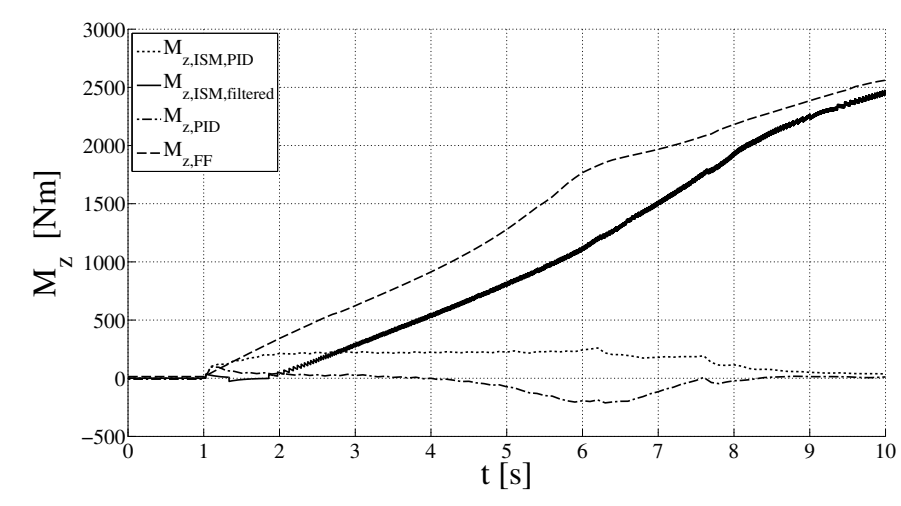

Figure 5 demonstrates that the ISM controller keeps the system permanently in sliding conditions as the amplitude of the oscillations around the manifold $s$ is very low (between 0.1 and $0.2 \mathrm{deg} / \mathrm{s}$ ). The yaw rate error, $-\Delta r$, during the manoeuvre is negligible for both controllers and rather significant for the passive vehicle. The corresponding yaw moment contributions from the different controllers are shown in Figure 6. Interestingly, the filtered yaw moment contribution of the ISM controller, $M_{z, I S M \text {,filtered }}$, is very similar to the feedforward contribution of the PID FF controller, while the PID contributions are low for both the ISM and the PID FF controllers. This important observation means that the ISM not only works as a disturbance observer, as expected from the theory, but in this application the ISM is also the main element responsible for achieving the steady-state yaw rate tracking performance. The ISM can thus potentially replace the FF element of a more conventional torque-vectoring controller, reducing the time and costs of control tuning.

\subsection{Sequence of step steers in high friction conditions}

Figure 7 reports the time history of the steering wheel angle, $\delta$, and yaw rate error, $-\Delta r$, for the passive vehicle, the vehicle with the ISM and the vehicle with the PID FF controller. Both the ISM and the PID FF reduce the oscillations of the yaw rate error, characterised by the typical dynamics of an underdamped second order system. The ISM allows a considerable reduction in the yaw rate error peaks following the completion of each steering wheel input.

Figure 8 confirms the significant improvement in the yaw rate dynamics induced by the ISM; the overshoots of $r$ are eliminated and the amplitudes of the yaw rate oscillations are greatly reduced. Consequently, the peak values of $\beta$ are significantly lower for the ISM than for the combined PID FF controller. The controlled vehicle is characterised by higher values of steady-state yaw rate than the passive vehicle, as specified by the respective understeer characteristics. 
Figure $7 \delta(t)$ (upper) and $-\Delta r(t)$ (lower) for a sequence of step steers
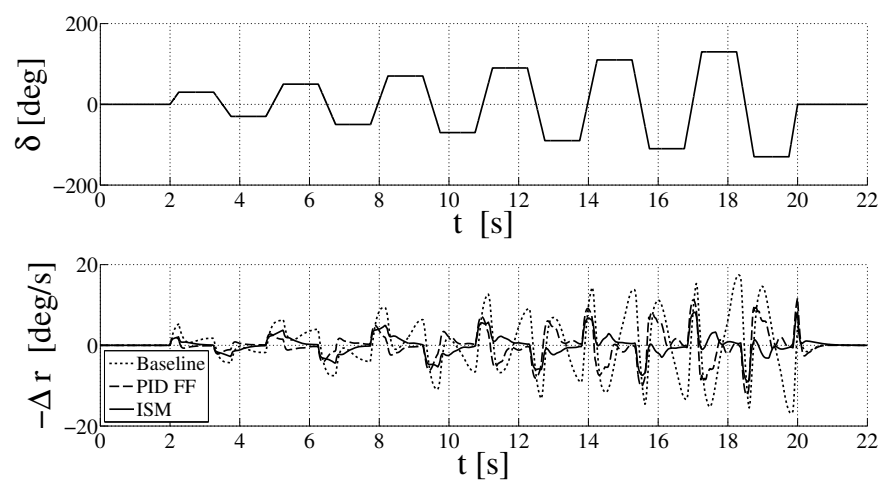

Figure $8 \quad r(t)$ (higher) and $\beta(t)$ (lower) for a sequence of step steers
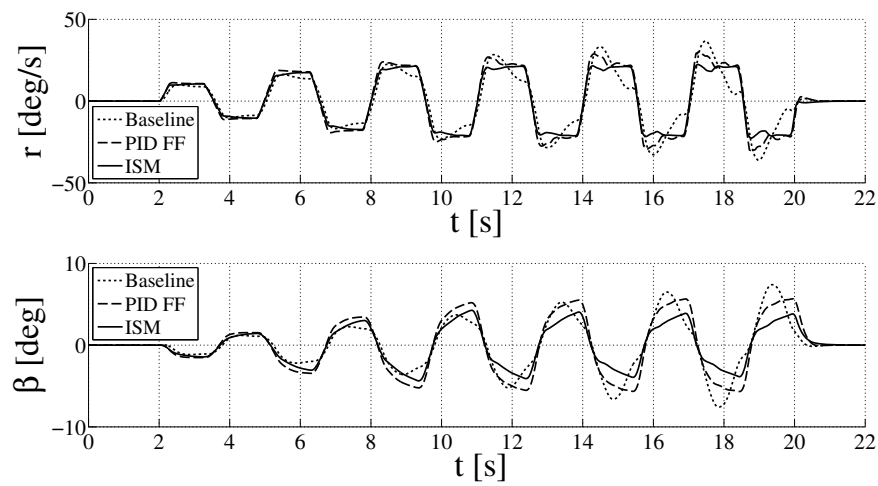

Figure 9 ISM yaw moment contributions for a sequence of step steers

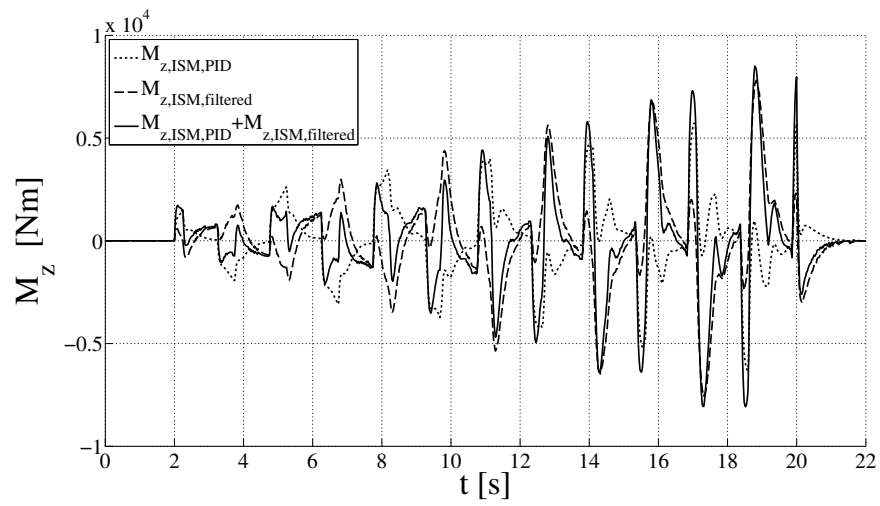

Figure 9 shows the smoothness of the ISM control action compared to the combined PID FF controller in Figure 10. In fact, the feedforward contribution of the PID FF controller, being tuned for steady-state cornering conditions, tends to produce a destabilising yaw moment during transients and when the steering wheel input is interrupted. Subsequently, when the yaw rate overshoot builds up at constant steering wheel angle, the PID 
contribution becomes stabilising and can reach very significant values. The peak values of $M_{z, P I D}$ are actually reached in this phase and the profile of $M_{z, I S M, P I D}$ is much smoother than that of $M_{z, P I D}$. This observation confirms that the performance of the ISM greatly exceeds that of a disturbance observer also during extreme transients. Even if the feedforward contribution of the PID FF were tuned to simultaneously increase the yaw damping during transients in addition to increasing the steady-state yaw rate as described in De Novellis et al. (2015), this would be a very difficult task to accomplish in practice, due to the nonlinear dynamic response of the vehicle.

In order to analyse the robustness of the proposed controllers against variations in the vehicle parameters, the performance of each controller is reported for different values of the inertial parameters of the vehicle (i.e., $m$ and $J_{z}$ ) and tyre parameters. The lateral force characteristics of two tyre typologies suitable for the same case study vehicle are reported in Figure 11. Tyre A is the standard equipment tyre and is the one adopted during the control design phase, while tyre B is a more sport-oriented tyre. For completeness this analysis is reported also for the case of PID only (with the same gains as for the PID FF).

Figure 10 PID FF yaw moment contributions for a sequence of step steers

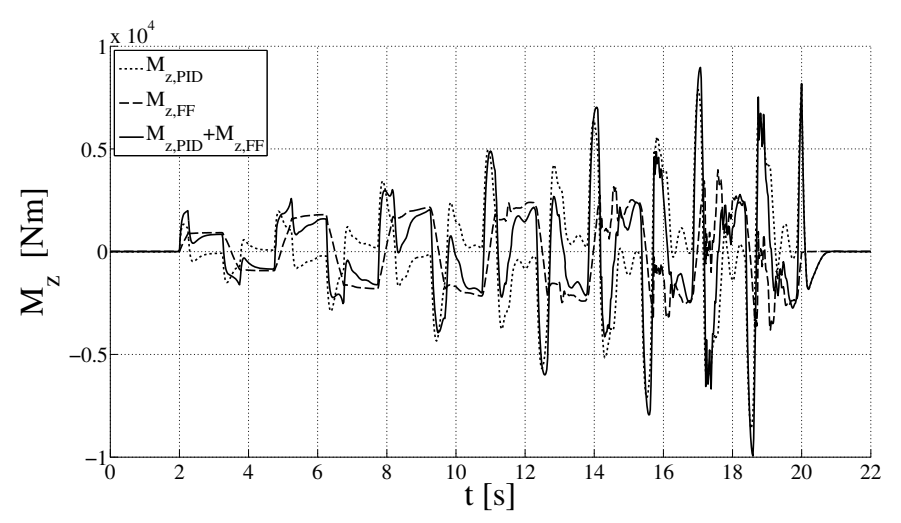

Figure 11 Comparison between tyre A (235/55 R19) and tyre B (255/35 R20): lateral tyre force as a function of slip angle for different vertical loads

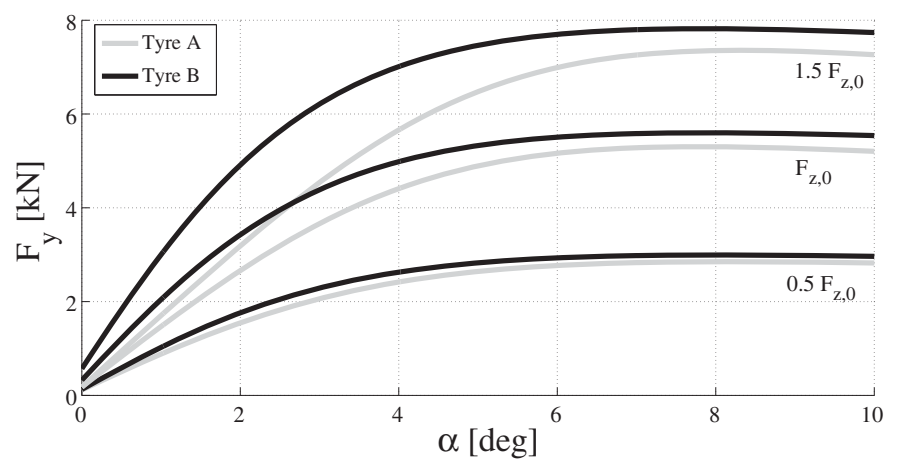

The $I A E$ values (Table 2) for the controlled vehicle are between $15 \%$ and $30 \%$ lower for the ISM controller than for the PID only controller, whilst the PID FF controller provides intermediate results. Interestingly, the ISM shows a consistently better performance than 
the PID and PID FF controllers for all conditions, though the variation in the IAE induced by the variation in the vehicle parameters is similar amongst the different controllers.

Table 2 IAE (integral of the absolute value of the yaw rate error) values [deg/s]

\begin{tabular}{lccccc}
\hline & $\begin{array}{c}m=1,797 \mathrm{~kg} \\
J_{z}=2,995 \mathrm{kgm}^{2}\end{array}$ & $\begin{array}{c}m=1,797 \mathrm{~kg} \\
J_{z}=2,995 \mathrm{kgm}^{2}\end{array}$ & & $\begin{array}{c}m=2,197 \mathrm{~kg} \\
J_{z}=3,662 \mathrm{kgm}^{2}\end{array}$ & $\begin{array}{c}m=2,197 \mathrm{~kg}^{2} \\
J_{z}=3,662 \mathrm{kgm}^{2}\end{array}$ \\
\cline { 2 - 3 } \cline { 2 - 3 } & Tyre A & Tyre B & & Tyre A & Tyre B \\
\hline PID & 2.33 & 3.12 & & 2.20 & 2.81 \\
PID FF & 2.03 & 2.62 & & 2.05 & 2.53 \\
ISM & 1.74 & 2.39 & & 1.90 & 2.29 \\
\hline
\end{tabular}

For tyre A, the ISM allows a significant reduction in the degree of control action as evaluated by the IACA; for example, the IACA with the ISM is reduced by about $20 \%$ with respect to the PID FF controller and more than $40 \%$ with respect to the PID controller for nominal operating conditions of the vehicle (Table 3). Table 4 shows the time delay between $r_{r e f}$ and $r$ for a couple of points along the same manoeuvre. ISM has the shortest time delay of vehicle response in comparison with the passive vehicle and the vehicle with a conventional continuous controller. This aspect is very important for exploiting the transient handling performance potential (including vehicle agility) of fully electric vehicles with multiple motors.

Table 3 IACA (integral of the absolute value of the control action) values [Nm]

\begin{tabular}{lccccc}
\hline & $\begin{array}{c}m=1,797 \mathrm{~kg} \\
J_{z}=2,995 \mathrm{kgm}^{2}\end{array}$ & $\begin{array}{c}m=1,797 \mathrm{~kg} \\
J_{z}=2,995 \mathrm{kgm}^{2}\end{array}$ & & $\begin{array}{c}m=2,197 \mathrm{~kg} \\
J_{z}=3,662 \mathrm{kgm}^{2}\end{array}$ & $\begin{array}{c}m=2,197 \mathrm{~kg}^{2} \\
J_{z}=3,662 \mathrm{kgm}^{2}\end{array}$ \\
\cline { 2 - 3 } \cline { 5 - 6 } & Tyre $A$ & Tyre $B$ & & Tyre $A$ & Tyre B \\
\hline PID & 2,301 & 2,586 & & 2,500 & 2,589 \\
PID FF & 1,912 & 2,235 & & 2,101 & 2,355 \\
ISM & 1,606 & 2,677 & & 1,855 & 2,540 \\
\hline
\end{tabular}

Table 4 Time delay between $r_{r e f}$ and $r[\mathrm{~ms}]$

\begin{tabular}{lcc}
\hline & $r_{r e f}=15 \mathrm{deg} / \mathrm{s}$ at $t=17.07 \mathrm{~s}$ & $r_{\text {ref }}=0 \mathrm{deg} / \mathrm{s}$ at $t=18.50 \mathrm{~s}$ \\
\hline Baseline & 110 & 73 \\
PID FF & 49 & 66 \\
ISM & 45 & 48 \\
\hline
\end{tabular}

Figure 12 shows a comparison between the yaw rate tracking performance of the ISM implementations of Case 1, Case 2 and Case 3 formulated in Section 3.2.2. The different formulations have a significant impact on the dynamic response of the vehicle, with the ISM configurations of Case 2 and Case 3 for the same tuning of the gain $K$ and time constant $\tau_{\text {contoller }}$ giving rise to an overshoot in $r(t)$ not present for Case 1 . 
Figure $12 r_{\text {ref }}$ reference yaw rate profile; $r_{1}$ refers to ISM Case 1, $r_{2}$ to ISM Case 2 and $r_{3}$ to ISM Case 3

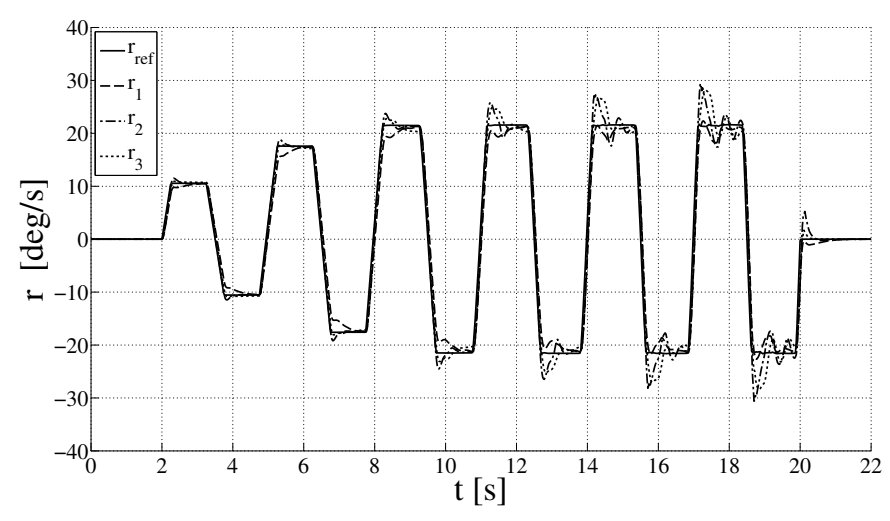

\subsection{Sine-with-dwell test}

The sine-with-dwell test, defined in both FMVSS 126 and ECE R13H, is characterised by a smooth steering wheel input, and at the same time offers the possibility of an objective and quantitative assessment of the vehicle response. The test evaluates whether the vehicle yaw rate swiftly returns back to low values following the completion of the specified steering wheel input, in order to ensure vehicle stability, and (in case of significant amplitudes of the steering wheel input) whether the lateral displacement of the vehicle is larger than the value provided by the specification, in order to ensure the required agility to prevent an impact with an obstacle.

Figure 13 reports the simulation results for the sine-with-dwell test with the baseline vehicle, which does not meet the requirements of FMVSS 126 for a steering wheel input amplitude of $4 \delta_{A}$, where $\delta_{A}$ is the steering wheel angle corresponding to a steady-state lateral acceleration of $0.3 \mathrm{~g}$ at the speed of $80 \mathrm{~km} / \mathrm{h}$. In fact, Figure 13 shows that the yaw rate value at $T_{0}+1 \mathrm{~s}$ (with $T_{0}$ being the time at which the steering wheel angle returns back to zero at the completion of the steering wheel angle application) is already beyond the limits specified by the regulation and that the yaw rate response is unable to follow the steering wheel input.

Figure $13 r(t)$ for the sine-with-dwell tests simulated for the baseline vehicle (i.e., without any controller) for different amplitudes of steering wheel input

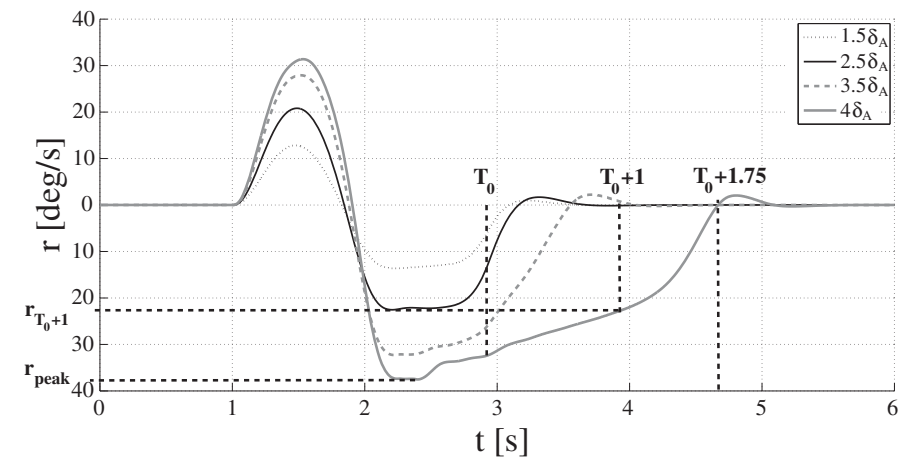


Figure $14 r(t)$ for the sine-with-dwell test simulated for the vehicle with the ISM controller for different amplitudes of steering wheel input

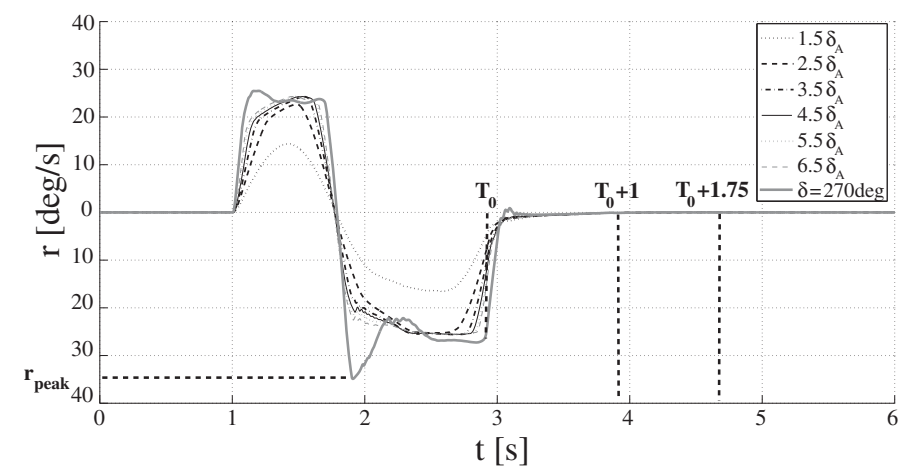

By way of contrast, the response of the controlled vehicle meets the requirements both in terms of yaw rate value (evaluated at $T_{0}+1 \mathrm{~s}$ and $T_{0}+1.75 \mathrm{~s}$, Figure 14) and lateral displacement (not reported here for brevity) with good margins for any relevant steering wheel angle amplitude. The value of $\delta_{A}$ is significantly lower for the ISM vehicle than for the baseline vehicle because of the different understeer characteristics.

\subsection{Sequence of step steers in low friction conditions}

Figures 15 and 16 confirm the excellent performance of the ISM controller in low friction conditions (a friction coefficient value of 0.3 ). The values of the gains of the PID within the ISM and the gains of the ISM are the same as for the high friction conditions. The passive vehicle is not capable of following the steering wheel inputs, as shown by the time histories of $r(t)$ and $\beta(t)$ after $11 \mathrm{~s}$ (condition of vehicle spin).

Figure $15 r(t)$ for the controlled vehicle and the passive vehicle (Baseline) during a sequence of step steers, with a friction coefficient of 0.3

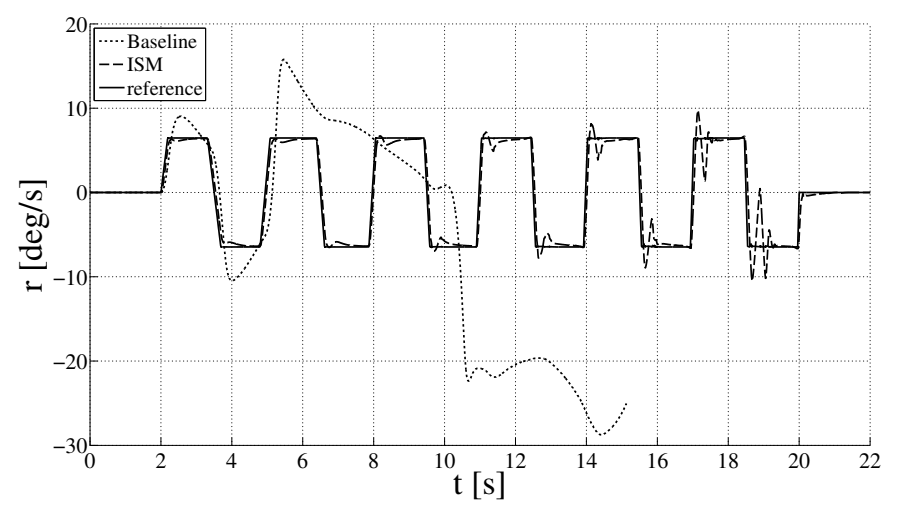


Figure $16 \beta(t)$ for the controlled vehicle and the passive vehicle (Baseline) during a sequence of step steers, with a friction coefficient of 0.3

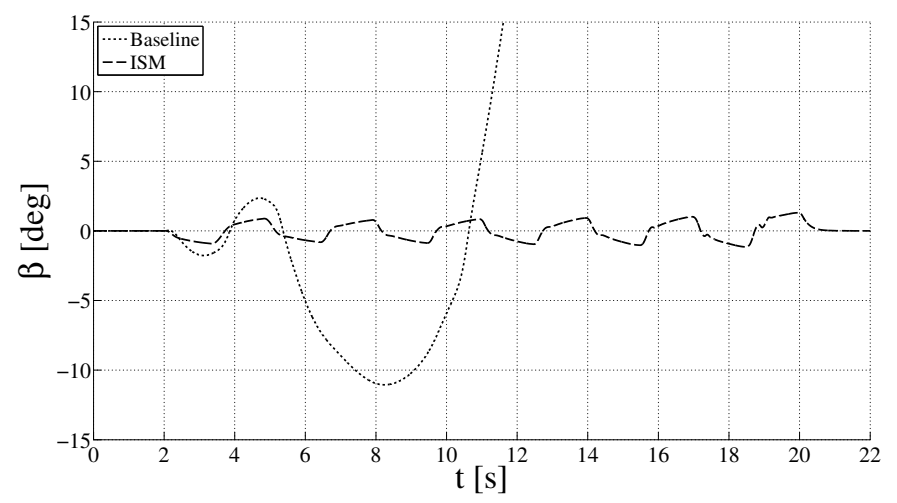

These results are obtained under the hypothesis that the low friction coefficient is identified by the controller, which reduces the reference value of yaw rate accordingly. The occurrence of less aggressive dynamics of the sideslip angle of the controlled vehicle for low friction than for high friction conditions is due to the fact that the tuning of the reference yaw rate is less aggressive in case of low $\mu$, which is considered a safety-critical condition.

\subsection{Sinusoidal yaw moment disturbance}

In Figures 17 and 18, the disturbance rejection properties of the ISM are evaluated. The ISM generates a yaw moment that compensates for most of the perturbation, unlike the PID FF controller, for which the amplitude of the resultant yaw rate oscillation is about four times greater than the amplitude of that of the vehicle with the ISM controller.

Figure 17 Yaw moment contributions of the PID FF controller (higher) and the ISM controller (lower) for a sinusoidal yaw moment disturbance $M_{z, \text { pert }}$

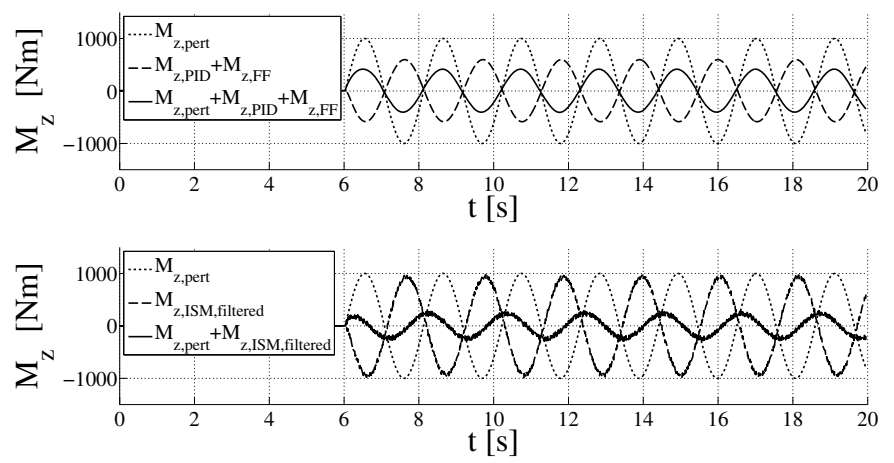


Figure $18 r(t)$ for a sinusoidal yaw moment disturbance $M_{z, p e r t}$

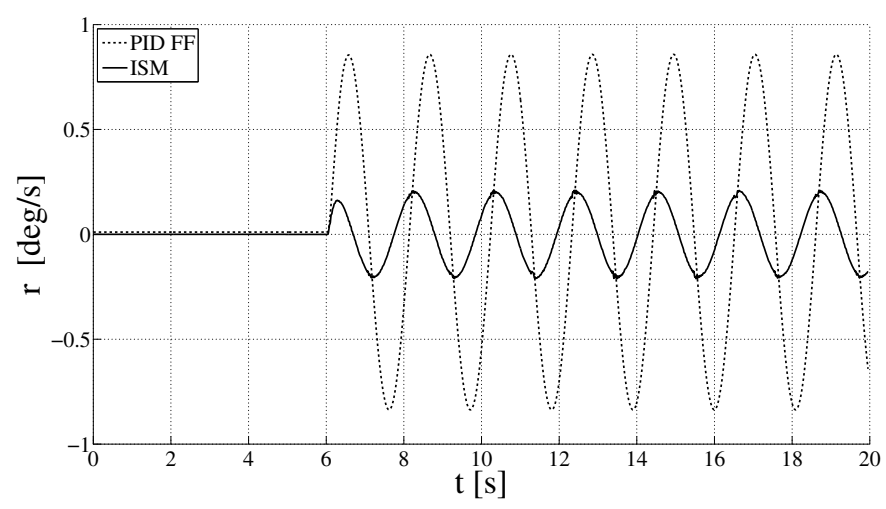

This analysis has been generalised in Figure 19, illustrating the frequency response characteristics of the ISM and PID FF controllers for different amplitudes of $M_{z, \text { pert }}$. The ISM coupled with the in-wheel drivetrains provides superior disturbance rejection, a capability which is a decreasing function of the disturbance frequency due to the first order filter adopted for smoothing $M_{z, I S M}$.

Figure 19 Frequency response characteristics (root mean square value of the yaw rate error, RMSE), evaluated for the ISM controller (continuous lines) and the PID FF controller (dashed lines), for sinusoidal yaw moment disturbances $M_{z, p e r t}$ at different amplitudes

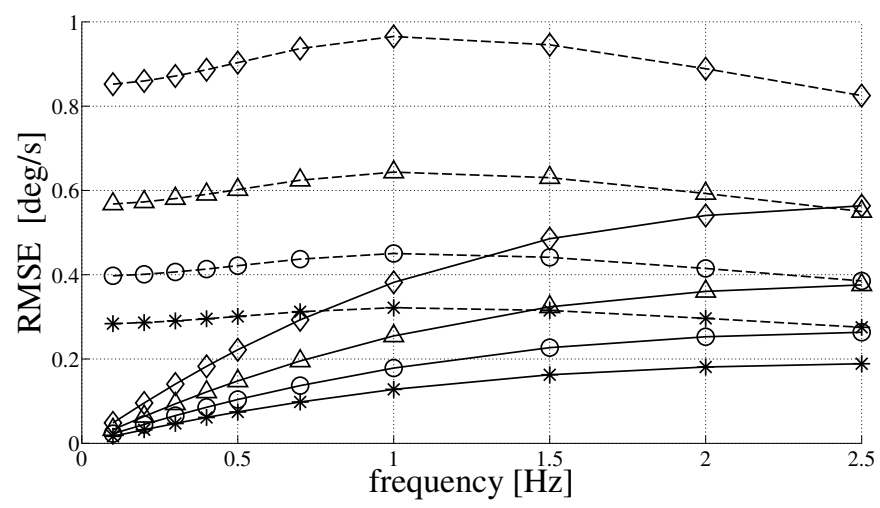

Notes: *: $500 \mathrm{Nm}$; ०: $700 \mathrm{Nm} ; \Delta: 1,000 \mathrm{Nm} ; \diamond: 1,500 \mathrm{Nm}$.

\subsection{Straight line manoeuvre with lateral wind gust}

Figure 20 shows the yaw rate response of the different controllers when a lateral wind gust is imposed. The ISM manages almost full compensation of the yaw rate disturbance; the peak amplitude of $r$ is $<0.4 \mathrm{deg} / \mathrm{s}$, and, thus, significantly lower than for the passive $(\sim 2.5 \mathrm{deg} / \mathrm{s})$ and the PID FF controlled $(\sim 1 \mathrm{deg} / \mathrm{s})$ vehicles. Note that in the case of the PID FF, only the PID part contributes to the reduction of the applied disturbance. 
Figure $20 r(t)$ during a lateral wind gust

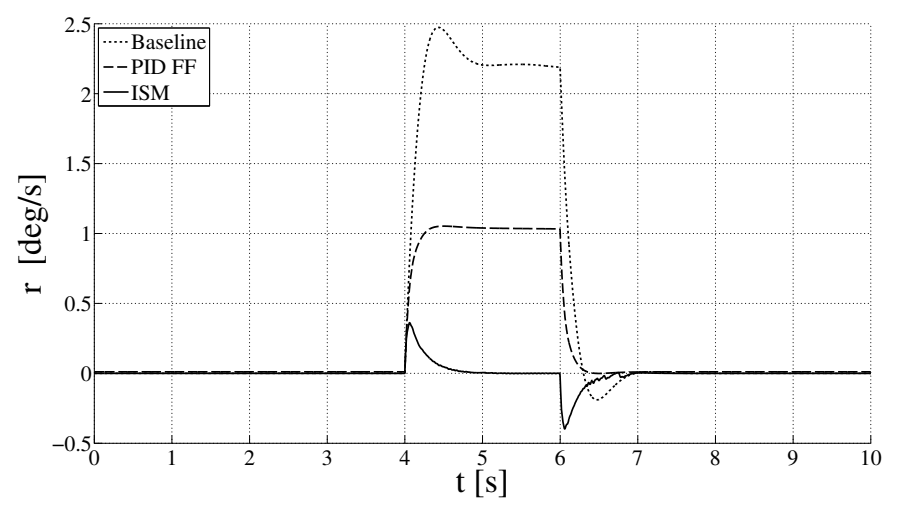

\subsection{Discussion}

The integral sliding mode controller presented in this paper guarantees a particularly good tracking performance of the reference yaw rate and robustness towards matched disturbances, with respect to the more conventional PID FF controller.

An aspect that will require further work is the analysis of the controller performance in case of measurement errors of the yaw rate sensor. As ISM is based on a feedback control structure (it actually includes a PID controller and the sliding variable is strictly related to the yaw rate error), if the yaw rate sensor is characterised by a measurement error, the actual yaw rate will also be subject to a corresponding error. In general, this phenomenon can be mitigated through the adoption of a controller including a significant feedforward contribution (mainly depending on the steering wheel angle and not on the yaw rate measurement) and a not particularly aggressive yaw rate feedback contribution. This is the main advantage of the PID FF controller adopted in the paper in comparison with the ISM. In case of significant concerns regarding the effect of the yaw rate error measurement, the FF contribution could be included in the ISM controller as well. Moreover, the effect of measurement noise on the ISM controller response will be investigated in future research.

\section{Conclusions}

An integral sliding mode controller for torque-vectoring control of a four-wheel-drive fully electric vehicle with in-wheel drivetrains has been presented. The ISM controller consistently improves the tracking performance, reduces control effort and compensates matched disturbances compared to a conventional PID FF controller, while the performance robustness against parameter uncertainty in the simulated test cases is comparable between ISM and the conventional controller. The feedforward action is not required for the ISM implementation, which considerably simplifies control system tuning and does not rely on extensive state estimation. In particular, the results of the ISM formulations of Case 1 and Case 3 show that the absence of state estimation does not adversely affect the control system performance during extreme transients. The filtering of the discontinuous part of the control action, intrinsic to the ISM formulation, avoids 
the chattering and vibration problems that commonly impede sliding mode implementations. Interestingly, the performance of the ISM for the specific application goes beyond its usual role of disturbance observer, but actually provides a significant contribution in nominal conditions. In fact, the yaw moment profile of the ISM during ramp steer manoeuvres is shown to be very similar to that of the nonlinear FF contribution of the PID FF controller.

Ongoing research is being carried out to analyse integral sliding mode for concurrent yaw rate and sideslip control, and gain scheduling for improving controller performance when evaluated on a wide range of operating conditions.

\section{Acknowledgements}

The research leading to these results has received funding from the European Union Seventh Framework Programme FP7/2007-2013 under Grant Agreement No. 284708 (E-VECTOORC project).

\section{References}

Ahn, C., Kim, B. and Lee, M. (2012) 'Modeling and control of an anti-lock brake and steering system for cooperative control on split-mu surfaces', International Journal of Automotive Technology, Vol. 13, No. 4, pp.571-581.

Ahn, C.S. (2011) Robust Estimation of Road Friction Coefficient for Vehicle Active Safety Systems, $\mathrm{PhD}$ thesis, University of Michigan.

Assadian, F. and Hancock, M. (2005) 'A comparison of yaw stability control strategies for the active differential', Proceedings of the IEEE International Symposium on Industrial Electronics.

Baffet, G., Charara, A. and Stephant, J. (2006) 'Sideslip angle, lateral tire force and road friction estimation in simulations and experiments', Proceedings of the IEEE International Conference on Control Applications.

Bottiglione, F., Sorniotti, A. and Shead, L. (2012) 'The effect of half-shaft torsion dynamics on the performance of a traction control system for electric vehicles', Proc. IMechE Part D: J Automobile Engineering, Vol. 226, No. 9, pp.1145-1159.

Canale, M., Fagiano, L., Ferrara, A. and Vecchio, C. (2005) 'Vehicle yaw control via second-order sliding-mode technique', IEEE Transactions on Industrial Electronics, Vol. 33, No. 11, pp.3908-3916.

Canale, M., Fagiano, L., Ferrara, A. and Vecchio, C. (2009) 'Comparing internal model control and sliding-mode', IEEE Transactions on Intelligent Transportation Systems, Vol. 5, Nos. 1-2, pp.31-41.

Chang, S. and Gordon, T. (2007) 'Model-based predictive control of vehicle dynamics', International Journal of Vehicle Autonomous Systems, Vol. 5, Nos. 1-2, pp.3-27.

Chen, Y. and Wang, J. (2010) 'Vehicle-longitudinal-motion-independent real-time tire-road friction coefficient estimation', 49th IEEE Conference on Decision and Control.

Chimner, C.T. and Grogg, J.A. (2013) Method and Apparatus for Real Time Estimation of Road Surface Friction Coefficient, US Patent 8,914,213.

Crolla, D.A. and Cao, D. (2012) 'The impact of hybrid and electric powertrains on vehicle dynamics, control systems and energy regeneration', Vehicle System Dynamics, Vol. 50, No. 1, pp.95-109. 
De Novellis, L., Sorniotti, A. and Gruber, P. (2013) 'Optimal wheel torque distribution for a four-wheel-drive fully electric vehicle', SAE International Journal of Passenger Cars, Vol. 6, No. 1, pp.128-136.

De Novellis, L., Sorniotti, A. and Gruber, P. (2014a) 'Design and comparison of the handling performance of different electric vehicle layouts', IMechE Part D: J Automobile Engineering, Vol. 228, No. 2, pp.218-232.

De Novellis, L., Sorniotti, A. and Gruber, P. (2014b) 'Wheel torque distribution criteria for electric vehicles with torque-vectoring differentials', IEEE Transactions on Vehicular Technology, Vol. 63, No. 4, pp.1593-1602.

De Novellis, L., Sorniotti, A., Gruber, P. and Pennycott, A. (2014c) 'Comparison of feedback control techniques for torque-vectoring control of fully electric vehicles', IEEE Transactions on Vehicular Technology, Vol. 63, No. 8, pp.3612-3623.

De Novellis, L., Sorniotti, A., Gruber, P., Ivanov, V. and Hoepping, K. (2012) 'Torque vectoring control for electric vehicles with individually controlled motors: state-of-the-art and future developments', Electric Vehicle Symposium, p.26.

De Novellis, L., Sorniotti, A., Gruber, P., Orus, J., Rodriguez Fortun, J.M., Theunissen, J. and De Smet, J. (2015) 'Direct yaw moment control actuated through electric drivetrains and friction brakes: theoretical design and experimental assessment', Mechatronics, Vol. 26, pp.1-15.

Eckert, A. (1998) System for Driving Stability Control, US Patent 5,774,821.

Fallah, S., Khajepour, A., Fidan, B., Chen, S.K. and Litkouhi, B. (2013) 'Vehicle optimal torque vectoring using state-derivative feedback and linear matrix inequality', IEEE Transactions on Vehicular Technology, Vol. 62, No. 4, pp.1540-1552.

Gaing, Z.L. (2004) 'A particle swarm optimization approach for optimum design of PID controller in AVR system', IEEE Transactions on Energy Conversion, Vol. 19, No. 2, pp.384-391.

Gao, L., Liu, S. and Dougal, R.A. (2002) 'Dynamic lithium-ion battery model for system simulation', IEEE Transactions on Components and Packaging Technologies, Vol. 25, No. 3, pp.495-505.

Gao, Y., Gray, A., Tseng, E.H. and Borrelli, F. (2014) 'A tube-based robust nonlinear predictive control approach to semiautonomous ground vehicles', Vehicle System Dynamics, Vol. 52, No. 6, pp.802-823.

Genta, G. (1997) Motor Vehicle Dynamics: Modelling and Simulation, World Scientific Publishin, Singapore.

Hahn, J.O., Rajamani, R. and Alexander, L. (2002) 'GPS-based real-time identification of tire-road friction coefficient', IEEE Transactions on Control Systems Technology, Vol. 18, No. 3, pp.331-343.

Hartmann, U. (2004) Method and Device for Situation-Dependent and Driver-Dependent Attenuation of ESP Stabilization Measures, US Patent 6,757,605 B2.

He, T., Ashrafi, B., Madau, D., Brown, T.A. and Recker, D. (1999) 'The development of vehicle stability control at Ford', IEEE/ASME Transactions on Mechatronics, Vol. 4, No. 3, pp. $223-234$.

Hsu, Y.J., Laws, S.M. and Gerdes, J.C. (2010) 'Estimation of tire slip angle and friction limits using steering torque', IEEE Transactions on Control Systems Technology, Vol. 18, No. 4, pp.896-907.

http://www.e-vectoorc.eu (accessed 28 July 2014).

IPG Automotive (2015) CarMaker [online] http://ipg.de/ (accessed 25 January 2015).

ISO 4138:2012 (2012) Passenger Cars - Steady-State Circular Driving Behaviour - Open-Loop Test Methods, International Organization for Standardization, Geneva.

ISO 7401:2011 (2011) Road Vehicles - Lateral Transient Response Test Methods - Open-Loop Test Methods, International Organization for Standardization, Geneva. 
Kaiser, G., Korte, M., Liu, Q., Hoffmann, C. and Werner, H. (2014) 'LPV torque vectoring for an electric vehicle with experimental validation', Proceedings of the 19th IFAC World Congress.

Lin, L.H., Wang, F.C. and Yen, J.Y. (2009) 'Robust PID controller design using particle swarm optimization', Proceedings of the 7th Asian Control Conference, Hong Kong.

Milliken, W.F. and Milliken, D.L. (1995) Race Car Vehicle Dynamics, SAE International, Warrendale, PA, USA.

Muller, S., Uchanski, M. and Hedrick, K. (2003) 'Estimation of the maximum tire-road friction coefficient', Journal of Dynamic Systems, Measurement and Control, Vol. 125, No. 4, pp.607-617.

Naraghi, M., Roshanbin, A. and Tavasoli, A. (2010) 'Vehicle stability enhancement - an adaptive optimal approach to the distribution of tyre forces', Proc IMechE Part D: J Automobile Engineering, Vol. 224, No. 4, pp.443-453.

National Highway Traffic Safety Administration FMVSS 126 (2007) Electronic Stability Control Systems, Final Regulatory Impact Analysis.

Pacejka, H.B. (2006) Tyre and Vehicle Dynamics, 2nd ed., Butterworth-Heinemann, Oxford.

Pastor, S.L. et al. (1998) Brake Control System, US Patent 5,720,533.

Pennycott, A., De Novellis, L., Sorniotti, A. and Gruber, P. (2014) 'The application of control and wheel torque allocation techniques to driving modes for fully electric vehicles', $S A E$ International Journal of Passenger Cars - Mechanical Systems, Vol. 7, No. 2, pp.488-496.

Ray, L.R. (1997) 'Nonlinear tire force estimation and road friction identification: simulation and experiments', Automatica, Vol. 33, No. 10, pp.1819-1833.

Shim, T. and Margolis, D. (2004) 'Model-based friction estimation', Vehicle System Dynamics, Vol. 41, No. 4, pp.249-276.

Takahashi, J., Yamakado, M., Saito, S. and Yokoyama, A. (2012) 'A hybrid stability-control system: combining direct-yaw moment control and G-vectoring control', Vehicle Systems Dynamics, Vol. 50, No. 6, pp.847-859.

Tanaka, S., Kawamoto, M. and Inagaki, H. (1992) Regenerative Braking Electric Vehicle with Four Motors, US Patent 5,148,883.

Tjonnas, J. and Johansen, T.A. (2010) 'Stabilization of automotive vehicles using active steering and adaptive brake control allocation', IEEE Transactions on Control System Technology, Vol. 18, No. 3, pp.545-558.

Utkin, V. and Shi, J. (1996) 'Integral sliding mode in systems operating under uncertainty conditions', 35th IEEE Conference on Decision and Control.

Utkin, V., Guldner, J. and Shi, J. (1999) Sliding Mode Control in Electromechanical Systems, Taylor \& Francis, London.

van Zanten, A. (2000) Bosch ESP Systems: 5 Years of Experience, SAE Technical paper 2000-01-1633.

van Zanten, A., Erhardt, R. and Pfaff, G. (1995) VDC, the Vehicle Dynamics Control System of Bosch, SAE Technical paper 950749.

Xiong, L., Yu, Z., Wang, C., Yang, C. and Meng, Y. (2012) 'Vehicle dynamics control of four in-wheel motor drive electric vehicle using gain scheduling based on tyre cornering stiffness estimation', Vehicle System Dynamics, Vol. 50, No. 6, pp.831-846.

\section{Notations}

The subscripts ' $R F$ ', ' $L F$ ', ' $R R$ ' and ' $L R$ ' respectively refer to the right front, left front, right rear and left rear drivetrains. The subscripts ' $F$ ' and ' $R$ ' respectively refer to the front and rear axles. The subscript ' $M A X$ ' refers to the maximum value of the respective parameter. 
$a$

$a_{p p}$

$a_{x}, a_{y}, a_{y}^{*}$

$b$

$b_{p p}$

$f$

$F_{x}$

$F_{y}$

$F_{z, 0}$

$h$

$h_{C G}$

$I A C A$

$I A E$

$J_{z}$

$k$

K

$K_{b}$

$$
K_{U}^{\text {lin }}
$$

L

$L_{\text {rel }}$

$m$

$M_{s a}$

$M_{z}$

$M_{z, C T R L}$

$M_{z, F F}$ front semi-wheelbase

accelerator pedal position

vehicle longitudinal acceleration, lateral acceleration and maximum lateral acceleration in the linear region of the understeer characteristic

rear semi-wheelbase

brake pedal position

known function of the states of the system

longitudinal tyre force

lateral tyre force

vertical tyre force level used as reference for plotting the tyre force characteristics

uncertain contribution (unmodelled dynamics and disturbances) to $\dot{x}$

height of the centre of gravity

integral of the absolute value of control action (divided by the integration time)

integral of absolute value of the error (divided by the integration time)

yaw moment of inertia

yaw moment contribution due to the lateral tyre forces and self-aligning torques

gain of the discontinuous part of the ISM control action

ratio between the friction brake torque and the brake pressure for an individual friction brake (with different values on the front and rear axles)

understeer gradient in the linear part of the understeer characteristic

vehicle wheelbase

tyre relaxation length

vehicle mass

self-aligning tyre torque

generic yaw moment

reference yaw moment, saturated taking into account the constraints due to the electric drivetrain torques and the estimated average tyre-road friction coefficient

feedforward yaw moment contribution within the PID FF controller 


\begin{tabular}{|c|c|}
\hline$M_{z, I S M}$ & yaw moment contribution due to the discontinuous part of the ISM \\
\hline$M_{z, I S M, f i l t e r e d}$ & filtered value of $M_{z, I S M}$ \\
\hline$M_{z, I S M, P I D}$ & yaw moment contribution due to the PID controller within the ISM \\
\hline$M_{z, p e r t}$ & sinusoidal yaw moment (perturbation) \\
\hline$M_{z, P I D}$ & $\begin{array}{l}\text { yaw moment contribution due to the PID controller within the PID } \\
\text { FF controller }\end{array}$ \\
\hline$M_{z, T O T}$ & $\begin{array}{l}\text { yaw moment given by the sum of several contributions, still to be } \\
\text { saturated according to the drivetrain limitations and the estimated } \\
\text { average tyre-road friction coefficient }\end{array}$ \\
\hline$M_{z, \text { unc }}$ & $\begin{array}{l}\text { yaw moment contribution due to uncertainties (i.e., unmodelled } \\
\text { dynamics) and disturbances }\end{array}$ \\
\hline$n$ & $\begin{array}{l}\text { known contribution to } \dot{x} \text {, i.e., the factor (function of the states of the } \\
\text { system) to be multiplied by the system input }\end{array}$ \\
\hline$p$ & Laplace variable \\
\hline$p_{b}$ & $\begin{array}{l}\text { reference pressure for the friction brakes (with different values on } \\
\text { each vehicle corner) }\end{array}$ \\
\hline$P_{M}$ & drivetrain power \\
\hline$r, \dot{r}$ & yaw rate, yaw acceleration \\
\hline$r_{\text {peak }}$ & peak value of yaw rate during the sine-with-dwell test \\
\hline$r_{r e f, S}, r_{r e f}, \dot{r}_{r e f}$ & $\begin{array}{l}\text { steady-state value of reference yaw rate, reference yaw rate, } \\
\text { reference yaw acceleration }\end{array}$ \\
\hline$r_{1}, r_{2}, r_{3}$ & $\begin{array}{l}\text { actual yaw rate, respectively for ISM Case } 1 \text {, ISM Case } 2 \text { and } \\
\text { ISM Case } 3\end{array}$ \\
\hline$R_{w}$ & wheel radius \\
\hline$s$ & sliding variable \\
\hline$s_{0}$ & conventional part of the sliding variable (e.g., the yaw rate error) \\
\hline$t$ & time \\
\hline$t_{m a n, i n}, t_{m a n, f i n}$ & $\begin{array}{l}\text { initial and final times of the relevant part of the manoeuvre, i.e., the } \\
\text { one characterised by the steering wheel input }\end{array}$ \\
\hline$T$ & track width \\
\hline$T_{m}$ & reference electric motor torque \\
\hline$T_{w}$ & reference torque for each wheel \\
\hline$T_{w, t o t}$ & overall reference torque (total reference torque of the four wheels) \\
\hline$T_{w, t o t, m o d}$ & $\begin{array}{l}\text { overall reference torque (total reference torque of the four wheels) } \\
\text { modified by the yaw moment controller }\end{array}$ \\
\hline
\end{tabular}




\begin{tabular}{|c|c|}
\hline$T_{0}$ & $\begin{array}{l}\text { time at which the steering wheel angle goes back to } 0 \mathrm{deg} \text { at the } \\
\text { completion of the sine-with-dwell test }\end{array}$ \\
\hline$u$ & system input (controller output) \\
\hline$u_{0}$ & $\begin{array}{l}\text { continuous control action within the ISM, e.g., due to a PID } \\
\text { controller }\end{array}$ \\
\hline$u_{1}$ & discontinuous part of the ISM control action \\
\hline$u_{1, f i l t e r e d}, \dot{u}_{1, \text { filtered }}$ & $\begin{array}{l}\text { filtered component of the discontinuous ISM control action and its } \\
\text { time derivative }\end{array}$ \\
\hline$v$ & vehicle speed \\
\hline$x, \dot{x}$ & generic state variable and its time derivative \\
\hline$z, \dot{z}$ & $\begin{array}{l}\text { auxiliary part of the sliding variable, typical of ISM, and its time } \\
\text { derivative }\end{array}$ \\
\hline$a$ & slip angle of a tyre \\
\hline$\beta, \dot{\beta}$ & vehicle body sideslip angle and sideslip rate \\
\hline$\delta$ & steering wheel angle \\
\hline$\delta_{A}$ & $\begin{array}{l}\text { steering wheel angle corresponding to a lateral acceleration of } 0.3 \mathrm{~g} \\
\text { at } 80 \mathrm{~km} / \mathrm{h}\end{array}$ \\
\hline$\delta_{W}, \delta_{W, d y n}, \delta_{W, k i n}$ & $\begin{array}{l}\text { steering angle (at the wheel), dynamic steering angle (at the wheel) } \\
\text { and kinematic steering angle (at the wheel) }\end{array}$ \\
\hline$\Delta r, \Delta \dot{r}$ & opposite of the yaw rate error $\left(\Delta r=r-r_{r e f}\right)$ and its time derivative \\
\hline$\Delta r_{\text {threshold }}$ & $\begin{array}{l}\text { threshold of vehicle yaw rate error, beyond which the yaw moment } \\
\text { controller modifies the wheel torque demand }\end{array}$ \\
\hline$\mu$ & average estimated friction coefficient between tyres and road surface \\
\hline$\tau_{\text {controller }}$ & time constant of the first order ISM filter \\
\hline$\tau_{M, F}$ & $\begin{array}{l}\text { ratio between the reference yaw moment generated by the difference } \\
\text { between the front wheel torques and the total reference yaw moment }\end{array}$ \\
\hline$\tau_{r}$ & $\begin{array}{l}\text { cut-off frequency of the first order transfer function used for defining } \\
\text { the reference yaw rate }\end{array}$ \\
\hline$\tau_{\text {trans }}$ & gear ratio of the in-wheel drivetrains \\
\hline$\tau_{\text {tyre }}$ & time constant of the first order relaxation tyre model \\
\hline$\tau_{T, F}$ & $\begin{array}{l}\text { ratio between the reference wheel torque on the front axle and the } \\
\text { total reference wheel torque. }\end{array}$ \\
\hline
\end{tabular}

